Part of Journal of Research of the National Bureau of Standards, Volume 24, February 1940

\title{
FIRST SPECTRUM OF TIN
}

\author{
By William F. Meggers
}

\section{ABSTRACT}

The spectrum emitted by neutral tin atoms has been measured, compiled, and analyzed. Revised wavelengths and relative intensities are given for 378 lines, 341 of which were observed photographically (1697.59 to $12536.5 \mathrm{~A}$ ), and 37 of greater wavelengths (12788.2 to $24738.2 \mathrm{~A}$ ) were measured radiometrically by Randall and Wright. About 80 percent of these lines are classified as combinations of energy levels arising from $5 p^{2}, 5 p n s, 5 p n p$, $5 p n d$, and $5 p n f$ electron configurations. The average difference between observed and computed wave numbers for lines measured photographically is $0.09 \mathrm{~cm}^{-1}$. Four series of the type $5 p^{2}-5 p n s$ yield a mean absolute value of $59155 \mathrm{~cm}^{-1}$ for the ground level, $5 p^{2} \cdot{ }^{3} \mathrm{P}_{0}$, of neutral tin atoms. From this, the principal ionization potential of tin is calculated to be 7.297 volts.

\section{CONTENTS}

Page

I. Introduction

II. Experiments_._.

III. Results _.

1. Wavelengths of Sn I lines

2. Term structure of the Sn I spectrum

3. Spectral series and ionization potential

IV. References.

\section{INTRODUCTION}

Several years ago, when the Research Laboratory of the Eastman Kodak Co. first produced the $Q_{-}$, and later the $Z$-, type of sensitizing for infrared photography, the spectroscopy section of the National Bureau of Standards tested the new plates by exploring arc-emission spectra in regions which had never been photographed before. In the case of tin, many new lines were thus discovered, and about 30 lines which had been observed hitherto only with bolometers and thermopiles were now photographed and measured with greater accuracy. Upon the completion of a new description of the infrared spectrum of tin, the published term analyses were examined to see how many of the observed lines could be explained as combinations of known terms. It was disappointing to find that so simple a spectrum as that of tin had such an incomplete and inaccurate term analysis. Serious discrepancies among the data and interpretations of different workers arose apparently from unreliable descriptions, so it was decided to reobserve the entire spectrum with more care. The arc spectrum of pure tin was accordingly photographed and measured from its limit in the ultraviolet below $1700 \mathrm{~A}$ to wavelengths approaching $13000 \mathrm{~A}$ in the infrared. A preliminary account of this investigation was presented at a meeting of the Optical Society of America in February 
$1939[1],{ }^{1}$ and a complete report on this new description and analysis of the Sn I spectrum is the purpose of the present paper.

References to 81 publications on the spectra of tin previous to 1912 have been compiled and reviewed by $H$. Kayser [2]. In the 27 years which have elapsed since then, more than 60 papers dealing with tin spectra have appeared; but only those which are considered most relevant to the description and analysis of the first spectrum of tin will be mentioned here. It is interesting to note that the first extensive measurements of tin arc spectra were made by Kayser and Runge [3] in 1894. They measured the wavelengths of 73 tin arc lines (5631.91 to 2053.8 A) on Rowland's scale and discovered the first significant regularities in this spectrum. The arc spectrum of tin was first measured relative to international secondary standards in 1914 by Arnolds [4], who published wavelength values for 66 lines (6149.620 to $2194.42 \mathrm{~A}$ ). Data for 49 lines among longer waves (9146.3 to $5631.70 \mathrm{~A}$ ) were supplied in 1928 by Walters [5]. Infrared data for 24 lines (13022.0 to 8554.7 A) first observed with bolometers by Randa 1 [6] in 1911 were revised and extended to 45 lines (24740.0 to 9852.1 A), 20 years later, by Randall and Wright [7], who, working with an 80-ampere arc between a carbon rod and a tin pool, observed the spectrum with thermopiles and a large grating spectrometer.

Tin arc spectra in the extreme ultraviolet were studied by McLellan, Young, and McLay [8], who photographed a 2-ampere arc to $1885 \mathrm{~A}$ with a small quartz spectrograph and recorded arc reversals from 1911 to $1756 \mathrm{~A}$ with a fluorite spectrograph exposed to tin in a carbon arc operated with 20 to 25 amperes. They admitted that their wavelength measurements might contain errors of $\pm 0.3 \mathrm{~A}$. As a consequence, deviations of 10 or more wave numbers were tolerated in socalled "constant differences," and many spurious levels and false classifications resulted. These unreliable data have frequently been quoted and analyzed by others attempting to further the classification of tin lines, but to this date no one has published improved wavelengths for extreme ultraviolet arc lines of tin. The absorption spectrum of tin has been studied by many, but most thoroughly by Zumstein [9], who observed 53 absorption lines in the ultraviolet. However, the extreme ultraviolet wavelengths are quoted from McLennan et al. [8].

The Zeeman effect of tin lines has been observed by Back [10] and by Green and Loring [11]. The former observed 26 lines (2334 to $5632 \mathrm{~A})$ and the latter 33 lines (2269 to $5632 \mathrm{~A})$. These two sets of data are in excellent agreement; they are of utmost importance for making quantum identifications of certain spectral terms.

Further comments on publications dealing with tin spectra will be given below in connection with the discussion of my results.

\section{EXPERIMENTS}

Conventional arcs (and sparks) at atmospheric pressure were employed as light sources in making this new description of the Sn I spectrum. Arcs were operated on a d-c circuit with 220 volts applied potential, and with series resistance to regulate the current between 6 and 12 amperes. On account of the low melting point of tin $\left(232^{\circ} \mathrm{C}\right)$, it was not possible to use solid electrodes in this arc, so lumps of pure

\footnotetext{
1 Figures in brackets indicate the literature references at the end of this paper.
} 
tin were burned in an arc having a pure copper rod (6 $\mathrm{mm}$ in diam) as upper electrode and a pool of molten tin in a cupped lower electrode of copper (12 $\mathrm{mm}$ in diam). Throughout the visible and ultraviolet spectra to $2000 \mathrm{~A}$, comparison spark spectra of tin were made, so that any lines belonging to ionized atoms which might appear on the arc spectrograms could be recognized and removed from the final list of Sn I lines. These spark spectra were produced with solid tin electrodes in the secondary circuit of a transformer rated at 30,000 volts, with capacitors of $0.006 \mu \mathrm{f}$ in parallel. The extreme ultraviolet spectrograms were made at Princeton University by A. G. Shenstone, who used an arc of 1 to 4 amperes between water-cooled electrodes of pure tin, or tin versus copper, in an atmosphere of pure nitrogen.

Descriptions of spectrographs and spectral intervals investigated with each are summarized as follows: Shenstone recorded the interval 1400 to $2200 \mathrm{~A}$ on Schumann plates with a normal-incidence vacuum spectrograph [12] having a 2-m glass grating ruled 30,000 lines to the inch. The dispersion is $4.2 \mathrm{~A} / \mathrm{mm}$ and the definition and resolving power are superb. Nine exposures were made ranging in time from 20 minutes to $2 \frac{1}{2}$ hours.

At the National Bureau of Standards most of the spectrograms were made with stigmatic concave-grating spectrographs [13]. An aluminized Pyrex grating (22 ft in radius) ruled 30,000 lines per inch by R. W. Wood [14] was used in the first order from 2000 to $8200 \mathrm{~A}$, and in the second order from 2500 to $4300 \mathrm{~A}$. The dispersion in the second order averages $1 \mathrm{~A} / \mathrm{mm}$. The near infrared (7600 to $8900 \mathrm{~A}$ ) was observed with an aluminized speculum grating originally ruled by H. A. Rowland with 20,000 lines per inch. The scale of these spectrograms was $3.5 \mathrm{~A} / \mathrm{mm}$. The entire infrared photographically accessible (7600 to $13000 \mathrm{~A}$ ) was explored with a speculum grating ruled by J. A. Anderson; it has 7,500 lines per inch and gives a scale of $10 \mathrm{~A} / \mathrm{mm}$. A portion of the ultraviolet (2100 to $2700 \mathrm{~A}$ ) was also observed with a large quartz Littrow spectrograph with dispersion ranging from 0.4 $\mathrm{A} / \mathrm{mm}$ to $1.0 \mathrm{~A} / \mathrm{mm}$. Exposure times required to photograph the arc spectrum of tin varied from 1 or 2 minutes in the near ultraviolet to 4 hours for the longest waves recorded in the infrared.

Besides Schumann plates for recording the extreme ultraviolet, the following types of spectroscopic plates supplied by the Research Laboratory of the Eastman Kodak Co. [15] were used. Plates coated with No. 33 emulsion were employed in the range 2100 to $4800 \mathrm{~A}, \mathrm{~F}$ sensitized plates from 4500 to $6800 \mathrm{~A}, N$-sensitized from 6500 to $8200 \mathrm{~A}, R$ - $P$-sensitized from 7600 to $8900 \mathrm{~A}, Q$-sensitized from 8000 to $11000 \mathrm{~A}$, and $Z$-sensitized from 10000 to $13000 \mathrm{~A}$.

The wavelengths (2100 to $8600 \mathrm{~A}$ ) were obtained by interpolation between iron lines, the iron arc spectrum being photographed adjacent to tin on all spectrograms except those in the extreme ultraviolet which had sufficient lines of $\mathrm{Cu}, \mathrm{C}$, and $\mathrm{N}$ to fix the scale and corrections for each exposure. The wavelengths reported here are based on international secondary standards [16] or on interferometer values of iron and copper lines by Burns and Walters [17], supplemented in the extreme ultraviolet (1656 to $1930 \mathrm{~A}$ ) by values of $\mathrm{C}$ and $\mathrm{N}$ lines recommended as tentative standards by Boyce and Robinson [18]. In the infrared and ultraviolet, outside the present limits of international secondary standards (6700 to $2500 \mathrm{~A}$ ), interferometer values of iron lines measured at the National Bureau of 
Standards [19] were relied upon. The first-order grating spectrum of tin beyond $8600 \mathrm{~A}$ was usually derived from iron lines in the second or third order, doubling or trebling the interpolated values of the apparent wavelengths of tin lines. (See fig. 1.)

On the $\mathrm{Sn}-\mathrm{Cu}$ spectrograms made by Shenstone the $\mathrm{Cu}$ lines could be recognized by their enhancement at one electrode, and tin spark lines (Sn II) were likewise distinguished by enhancement at one end of the slit images. (See fig. 2.)

All spectrograms were measured, both direct and reversed, on comparators reading to $0.001 \mathrm{~mm}$, and all lines were observed on four to six spectrograms except the faintest, which were discarded unless seen on at least two exposures. The grating spectrograms were reduced on the assumption of linear dispersion, and the prism spectrograms by aid of the Hartmann dispersion formula, corrections on account of variable dispersion being determined in every case from standards. Even under the best conditions, it is difficult to determine the wavelengths of tin lines within $0.01 \mathrm{~A}$, because almost all of the line images are either hazy, wide, and unsymmetrical, or wide and more or less self-reversed. However, the agreement between different spectrographs and between successive spectrograms on each, and especially the analysis of spectral structure reported below, indicate that the goal of improving the description of the first spectrum of tin has been attained.

Portions of typical spectrograms are reproduced in figures 1 and 2.

\section{RESULTS}

\section{WAVELENGTHS OF Sn I LINES}

Data for 378 lines characteristic of neutral tin atoms are presented in table 1 , where wavelengths, relative intensities, vacuum wave numbers, term combinations of classified lines, and residuals ( $\nu_{\text {obs. }}-$ $v_{\text {cale. }}$ ) appear in successive columns. Wave numbers for lines between 2000 and 10000 A were taken from Kayser's Tabelle der Schwingungszahlen [20]. For longer waves the values were first corrected to vacuum by extrapolating the dispersion formula of Meggers and Peters [21] and then computing reciprocals. Since the wavelengths shorter than $2000 \mathrm{~A}$ are vacuum values, the corresponding wave numbers are direct reciprocals of the observed wavelengths. 


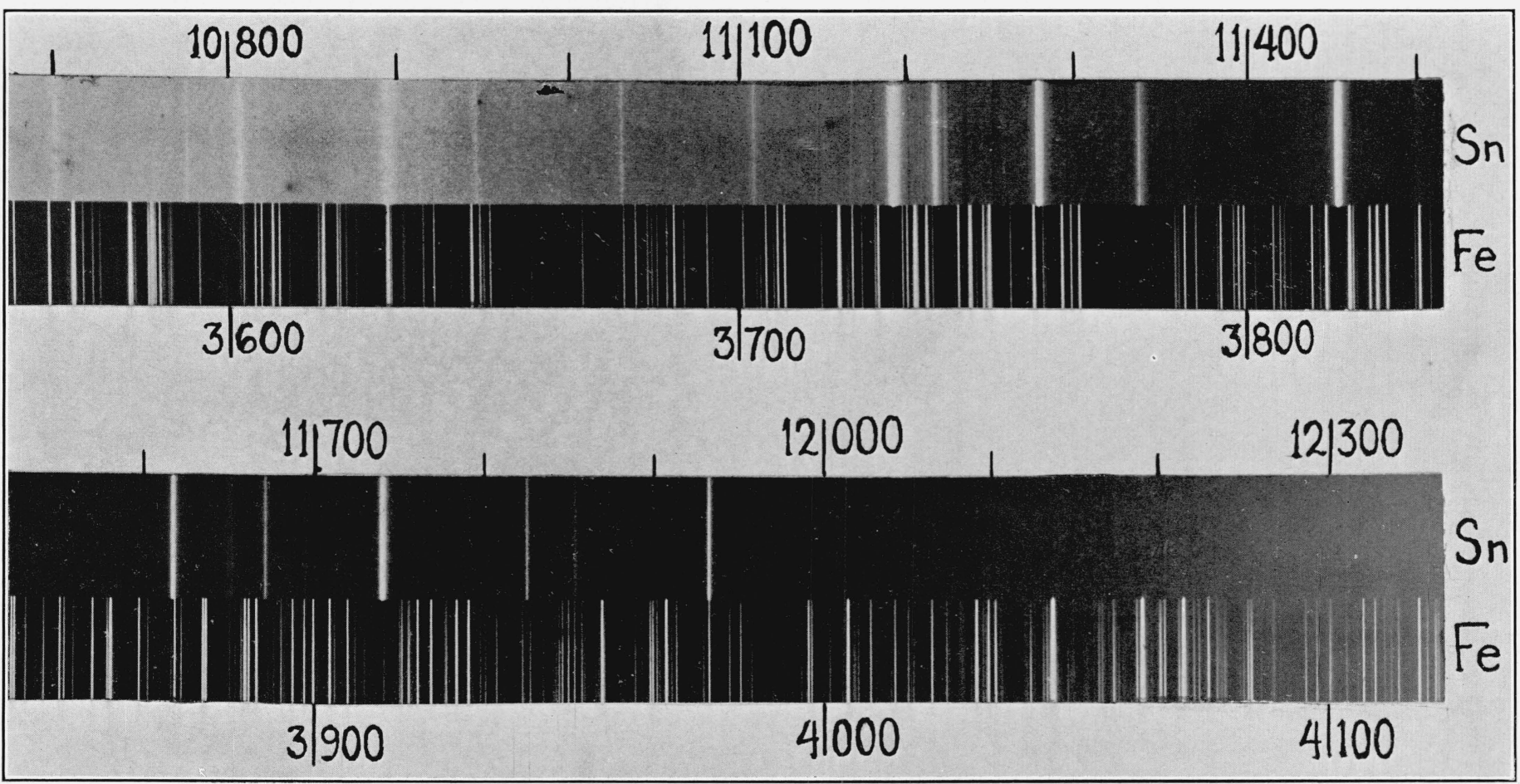

Figure 1.-Portion of infrared arc spectrum of tin. 


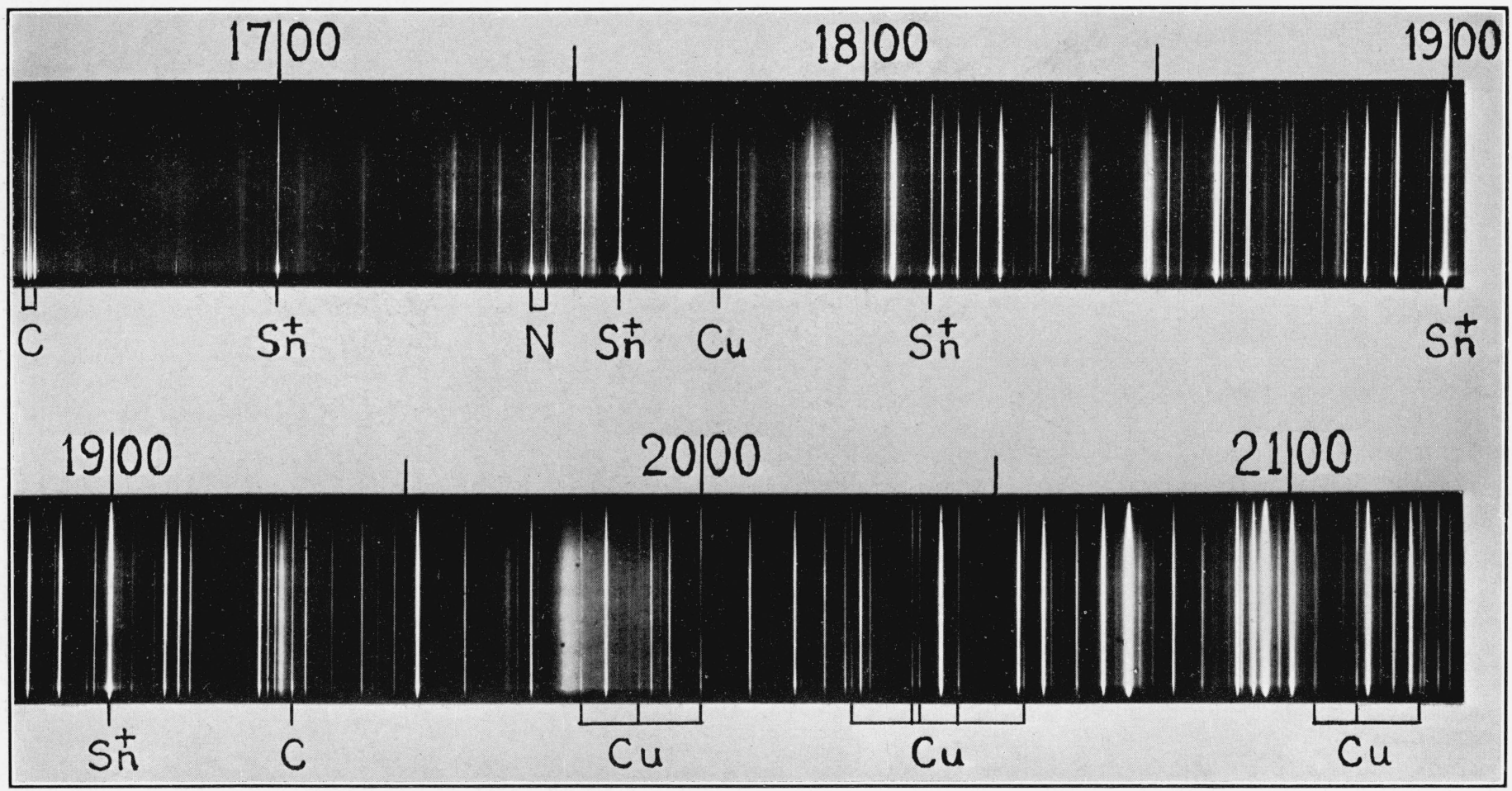

FIGURE 2.-Extreme ultraviolet arc spectrum of tin. 
TABLE 1.-First spectrum of tin (Sn I)

\begin{tabular}{|c|c|c|c|c|}
\hline$\lambda_{\mathrm{air}} \mathbf{A}$ & $\begin{array}{l}\text { Intensity and } \\
\text { character }\end{array}$ & $\nu_{\mathrm{VBc}} \mathrm{cm}^{-1}$ & Combination & $\begin{array}{c}\nu_{\mathrm{obs}}-\nu_{\mathrm{call}} \\
0 . \mathrm{i} \mathrm{cm}^{-1}\end{array}$ \\
\hline $\begin{array}{l}24738.2 \\
24327.2 \\
22997.2 \\
22131.7 \\
21686.2\end{array}$ & $\begin{array}{l}{[40]} \\
{[40]} \\
{[30]} \\
{[40]} \\
{[80]}\end{array}$ & $\begin{array}{l}4041.2 \\
4109.5 \\
4347.2 \\
4517.2 \\
4610.0\end{array}$ & $\begin{array}{c}6 s^{1} \mathrm{P}_{1}^{\circ}-6 p^{3} \mathrm{P}_{1} \\
6 p^{1} \mathrm{D}_{2}-7 s^{1} \mathrm{P}_{1}^{\circ} \\
6 p^{3} \mathrm{~S}_{1}-7 s^{3} \mathrm{P}_{2}^{\circ}\end{array}$ & $\begin{array}{r}-19 \\
+1 \\
0\end{array}$ \\
\hline $\begin{array}{l}\text { 20861. } 7 \\
20622.2 \\
20594.4 \\
17807.5 \\
17744.7\end{array}$ & $\begin{array}{l}{[400]} \\
{[200]} \\
{[70]} \\
{[100]} \\
{[70]}\end{array}$ & $\begin{array}{l}4792.2 \\
4847.8 \\
4854.4 \\
\text { 5614. } 1 \\
\text { 5633. } 9\end{array}$ & $\begin{array}{l}6 p^{3} \mathrm{P}_{0}-7 s^{3} \mathrm{P}_{\mathrm{i}} \\
6 p^{3} \mathrm{P}_{1}-7 s^{3} \mathrm{P}_{0}^{0} \\
6 p^{3} \mathrm{P}_{1}-7 s^{3} \mathrm{P}_{1} \\
6 p^{3} \mathrm{P}_{1}-5 d^{3} \mathrm{P}_{1}^{\circ}\end{array}$ & $\begin{array}{l}+1 \\
+1 \\
+8 \\
+6\end{array}$ \\
\hline $\begin{array}{l}17198.8 \\
17019.9 \\
17000.5 \\
16381.2 \\
15793.7\end{array}$ & $\begin{array}{l}{[40]} \\
{[100]} \\
{[200]} \\
{[70]} \\
{[30]}\end{array}$ & $\begin{array}{l}5812.8 \\
5873.9 \\
5880.6 \\
6102.9 \\
6329.9\end{array}$ & $\begin{array}{l}6 p^{1} \mathrm{P}_{1}-7 s^{3} \mathrm{P}_{2}^{0} \\
6 p^{3} \mathrm{D}_{1}-7 s^{3} \mathrm{P}_{0}^{0} \\
6 p^{3} \mathrm{D}_{1}-7 s^{3} \mathrm{P}_{1}^{1} \\
6 p^{1} \mathrm{P}_{1}-7 s^{1} \mathrm{P}_{1}^{1}\end{array}$ & $\begin{array}{r}+5 \\
0 \\
+8 \\
-5\end{array}$ \\
\hline $\begin{array}{l}\text { 15749. } 7 \\
\text { 15635. } 2 \\
15584.2 \\
15464.2 \\
15367.2\end{array}$ & $\begin{array}{l}{[70]} \\
{[100]} \\
{[50]} \\
{[300]} \\
{[100]}\end{array}$ & $\begin{array}{l}6347.6 \\
6394.1 \\
6415.0 \\
6464.8 \\
6505.6\end{array}$ & $\begin{array}{c}6 p{ }^{1} \mathrm{P}_{1}-8 s^{3} \mathrm{P}_{1} \\
6 p{ }^{1} \mathrm{D}_{2}-6 d^{3} \mathrm{P}_{2}^{\circ} \\
5 d^{1} \mathrm{~F}_{3}^{\circ}-2754\end{array}$ & $\begin{array}{l}-22 \\
+11 \\
-13\end{array}$ \\
\hline $\begin{array}{l}\text { 15055. } 2 \\
15018.2 \\
14797.2 \\
14668.2 \\
14482.2\end{array}$ & $\begin{array}{l}{[100]} \\
{[130]} \\
{[60]} \\
{[100]} \\
{[100]}\end{array}$ & $\begin{array}{l}6640.4 \\
6656.8 \\
6756.2 \\
6815.6 \\
6903.1\end{array}$ & $\begin{array}{c}6 p^{3} \mathrm{D}_{1}-5 d^{3} \mathrm{P}_{1} \\
5 d^{1} \mathrm{~F}_{3}^{\circ}-2605 \\
6 p^{3} \mathrm{P}_{1}-5 d^{1} \mathrm{P}_{1}^{\circ}\end{array}$ & $\begin{array}{r}+7 \\
+6 \\
-12\end{array}$ \\
\hline $\begin{array}{l}\text { 13608. } 2 \\
13460.2 \\
13349.2 \\
13320.9 \\
13081.5\end{array}$ & $\begin{array}{l}{[1440]} \\
{[3780]} \\
{[30]} \\
{[70]} \\
{[675]}\end{array}$ & $\begin{array}{l}\text { 7346. } 5 \\
7427.3 \\
7489.1 \\
7505.0 \\
7642.3\end{array}$ & $\begin{array}{l}6 s^{1} \mathrm{P}_{1}-6 p^{1} \mathrm{P}_{1} \\
6 s^{3} \mathrm{P}_{1}^{\circ}-6 p^{3} \mathrm{D}_{1} \\
6 p^{3} \mathrm{~S}_{1}-6 d^{1} \mathrm{D}_{2}^{\circ} \\
5 d^{3} \mathrm{P}_{1}^{\circ}-2668 \\
6 p^{3} \mathrm{P}_{1}-6 d^{3} \mathrm{D}_{2}^{\circ}\end{array}$ & $\begin{array}{r}+2 \\
-8 \\
-13 \\
+5 \\
+3\end{array}$ \\
\hline $\begin{array}{l}13018.5 \\
13000.3 \\
12981.7 \\
12934.2 \\
12888.5\end{array}$ & $\begin{array}{l}{[18 \% 0]} \\
{[200]} \\
{[18 \% 0]} \\
{[30]} \\
{[890]}\end{array}$ & $\begin{array}{l}\text { 7679. } 2 \\
7690.0 \\
7701.0 \\
7729.3 \\
7756.7\end{array}$ & $\begin{array}{c}6 p^{1} \mathrm{I}_{0}-6 s^{1} \mathrm{P}_{1} \cdot ? \\
5 d^{3} \mathrm{~F}_{3}^{\circ}-6891 \\
6 s^{3} \mathrm{P}_{0}^{\circ}-6 p^{3} \mathrm{D}_{1} \\
5 d^{3} \mathrm{D}_{1}-6891\end{array}$ & $\begin{array}{r}0 \\
+24 \\
-5 \\
+17\end{array}$ \\
\hline $\begin{array}{l}12845.2 \\
12788.2 \\
12536.5 \\
12530.87 \\
12335.6\end{array}$ & $\begin{array}{r}{[160]} \\
{[370]} \\
1[425] \\
1[425] \\
2[330]\end{array}$ & $\begin{array}{l}7782.9 \\
7817.6 \\
7974.5 \\
7978.11 \\
8104.4\end{array}$ & $\begin{array}{c}6 p^{3} \mathrm{D}_{1}-5 d^{1} \mathrm{P}_{1}^{\circ} \\
5 d^{3} \mathrm{P}_{2}^{\circ}-2668 \\
6 s^{3} \mathrm{P}_{2}^{\circ}-6 p^{1} \mathrm{P}_{1} \\
6 s^{1} \mathrm{P}_{1}^{\circ}-6 p^{3} \mathrm{P}_{2} \\
5 d^{3} \mathrm{~F}_{2}^{\circ}-6906\end{array}$ & $\begin{array}{r}-7 \\
+6 \\
-1 \\
0 \\
0\end{array}$ \\
\hline $\begin{array}{l}\text { 12313. } 24 \\
12054.5\end{array}$ & $\begin{array}{l}4[1110] \\
1[30]\end{array}$ & $\begin{array}{l}8119.11 \\
8293.4\end{array}$ & $5 d^{3} \mathrm{~F}_{2}^{\circ}-6891$ & 0 \\
\hline 12009.50 & $3[480]$ & 8324.47 & $\begin{array}{l}6 s^{3} \mathrm{P}_{1}-6 p^{3} \mathrm{D}_{2} \\
6 p^{3} \mathrm{P}_{0}-6 d^{3} \mathrm{~F}_{3}^{2}\end{array}$ & $\begin{array}{l}0 \\
0\end{array}$ \\
\hline $\begin{array}{l}11932.99 \\
11835.82\end{array}$ & $\begin{array}{r}30[2540] \\
4[1060]\end{array}$ & $\begin{array}{l}8377.84 \\
8433.79\end{array}$ & $6 s^{3} \mathrm{P}_{2}^{0}-6 p^{3} \mathrm{D}^{3}$ & -2 \\
\hline $\begin{array}{l}\text { 11848. } 04 \\
11825.18\end{array}$ & $\stackrel{2}{10[960]}$ & $\begin{array}{l}\text { 8437. } 91 \\
8454.22\end{array}$ & $\begin{array}{l}6 p^{3} \mathrm{~S}_{1}-2911 \\
6 s^{3} \mathrm{P}_{1}-6 p^{3} \mathrm{P}_{1}\end{array}$ & $\begin{array}{l}-1 \\
-1\end{array}$ \\
\hline 11739.78 & $80[2580]$ & 8515. 72 & $\begin{array}{r}6 s^{3} \mathrm{P}_{1}^{\circ}-6 p^{3} \mathrm{P}_{0} \\
6 p^{3} \mathrm{D}_{2}-6 d^{3} \mathrm{~F}_{3}^{0}\end{array}$ & $\begin{array}{l}-1 \\
+1\end{array}$ \\
\hline $\begin{array}{l}11694.45 \\
11670.77\end{array}$ & $\begin{array}{r}3[250] \\
10[760]\end{array}$ & $\begin{array}{l}8548.72 \\
8566.07\end{array}$ & $\begin{array}{c}6 s{ }^{1} \mathrm{P}_{1}-6 p{ }^{3} \mathrm{~S}_{1} \\
5 d^{3} \mathrm{D}_{2}^{\circ}-6906\end{array}$ & $\begin{array}{l}+1 \\
+1\end{array}$ \\
\hline $\begin{array}{l}11650.76 \\
11648.32 \\
11616.26 \\
11533.4 \\
11500.9\end{array}$ & $\begin{array}{l}3[125] \\
2 \\
60[2000] \\
2 h[30] \\
2 h\end{array}$ & $\begin{array}{l}8580.78 \\
8582.58 \\
8606.27 \\
8668.1 \\
8692.6\end{array}$ & $\begin{array}{l}5 d^{3} \mathrm{D}_{2}^{\circ}-6891 \\
6 s^{3} \mathrm{P}_{2}^{\circ}-6 p^{3} \mathrm{P}_{2} \\
6 p^{3} \mathrm{D}_{1}-6 d^{3} \mathrm{D}_{2}^{\circ} \\
6 p^{1} \mathrm{P}_{1}-6 d^{1} \mathrm{D}_{2}^{\circ}\end{array}$ & $\begin{array}{r}0 \\
-1 \\
-1 \\
-1\end{array}$ \\
\hline
\end{tabular}


TABLE 1.-First spectrum of tin ( $\mathrm{Sn} \mathrm{I})$-Continued

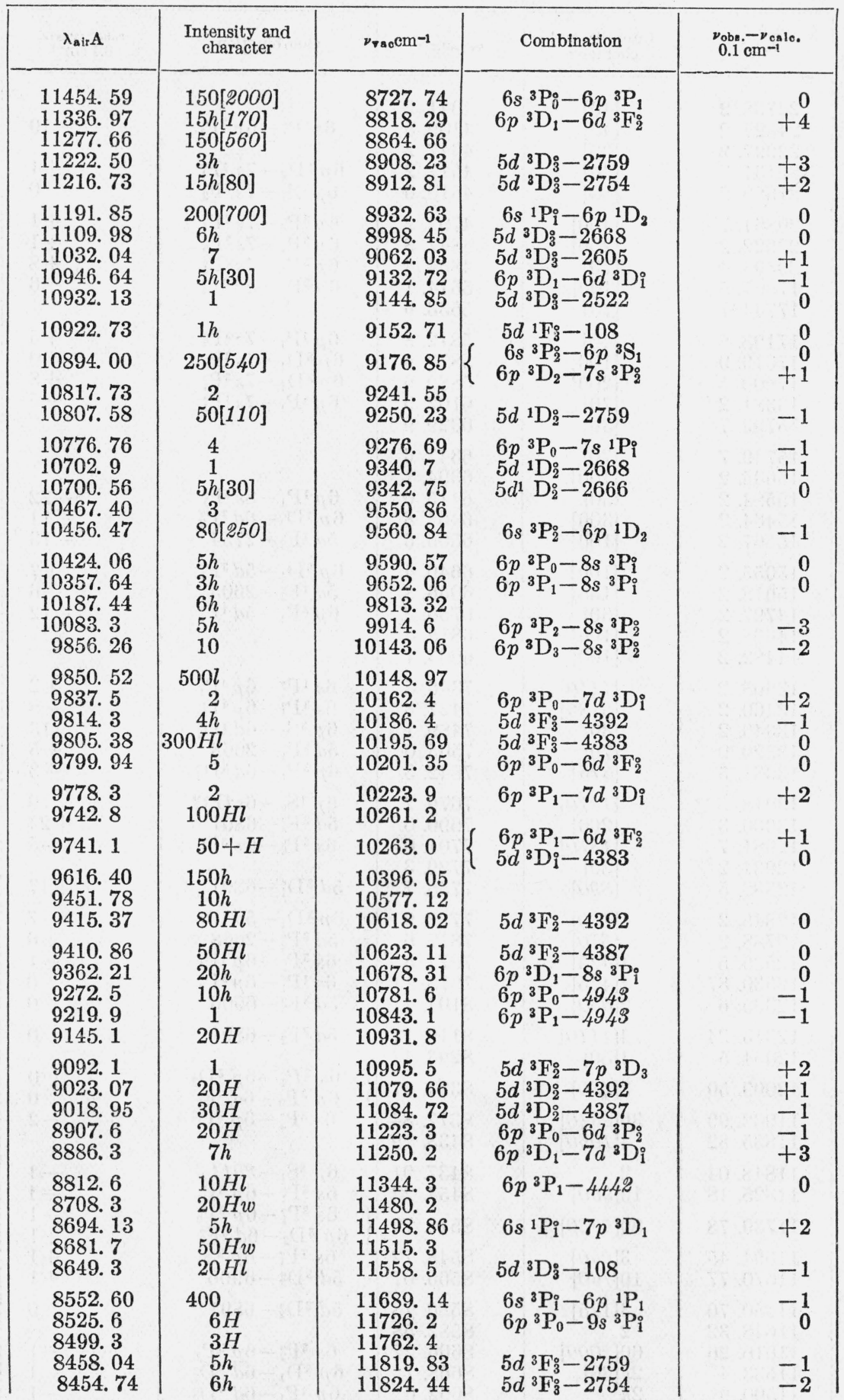


TABLE 1.-First spectrum of tin (Sn I)-Continued

\begin{tabular}{|c|c|c|c|c|}
\hline$\lambda_{\mathrm{Bir}} \mathbf{A}$ & $\begin{array}{l}\text { Intensity and } \\
\text { character }\end{array}$ & $\nu_{\mathrm{B}} \mathrm{c} \mathrm{cm}^{-1}$ & Combination & $\begin{array}{l}\nu_{\text {oba }}-\nu_{\text {eale. }} \\
0.1 \mathrm{~cm}^{-1}\end{array}$ \\
\hline $\begin{array}{l}8432.14 \\
8422.72 \\
8393.83 \\
8391.31 \\
8381.69\end{array}$ & $\begin{array}{c}5 h \\
300 h l \\
4 h \\
20 h \\
4 h\end{array}$ & $\begin{array}{l}\text { 11856. } 13 \\
11869.39 \\
11910.24 \\
11913.82 \\
11927.49\end{array}$ & $\begin{array}{l}6 s^{1} \mathrm{P}_{1}-7 p^{3} \mathrm{P}_{1} \\
6 p^{3} \mathrm{D}_{1}-4943^{3} \\
5 d^{3} \mathrm{~F}_{3}^{\circ}-2668 \\
6 s^{1} \mathrm{P}_{1}^{\circ}-7 p^{3} \mathrm{D}_{2} \\
6 p^{3} \mathrm{P}_{1}-6 d^{1} \mathrm{D}_{2}^{\circ}\end{array}$ & $\begin{array}{r}-1 \\
0 \\
-2 \\
+1 \\
-1\end{array}$ \\
\hline $\begin{array}{l}8357.04 \\
8349.35 \\
8345.23 \\
8338.6 \\
8250.03\end{array}$ & $\begin{array}{l}80 \\
30 h \\
10 h \\
10 H w \\
8 h\end{array}$ & $\begin{array}{l}\text { 11962. } 68 \\
11973.70 \\
11979.60 \\
11989.1 \\
12117.84\end{array}$ & $\begin{array}{c}6 s^{3} \mathrm{P}_{0}^{\circ}-6 p{ }^{1} \mathrm{P}_{1} \\
5 d^{3} \mathrm{~F}_{3}^{\circ}-2605 \\
5 d^{3} \mathrm{D}_{1}^{\circ}-2666 \\
{ }^{1} \mathrm{P}_{1}-7 p^{3} \mathrm{P}_{0}\end{array}$ & $\begin{array}{l}+1 \\
-2 \\
-1 \\
+1\end{array}$ \\
\hline $\begin{array}{l}8247.22 \\
8245.84 \\
8243.8 \\
8160.08 \\
8133.2\end{array}$ & $\begin{array}{c}2 h \\
4 h \\
1 h \\
2 h \\
10 H w\end{array}$ & $\begin{array}{l}\text { 12121. } 96 \\
\text { 12124. } 00 \\
12127.0 \\
12251.42 \\
12291.9\end{array}$ & $\begin{array}{l}5 d^{3} \mathrm{D}_{\mathrm{i}}-2522 \\
6 s^{3} \mathrm{P}_{2}^{\circ}-7 p^{3} \mathrm{D}_{1} \\
5 d^{3} \mathrm{~F}_{2}^{\circ}-2759\end{array}$ & $\begin{array}{r}-1 \\
0 \\
0\end{array}$ \\
\hline $\begin{array}{l}8121.0 \\
8114.09 \\
8100.25 \\
8046.8 \\
8039.3\end{array}$ & $\begin{array}{c}30 H l \\
200 \\
3 h \\
5 H l \\
30 H l\end{array}$ & $\begin{array}{l}12310.4 \\
12320.86 \\
12341.91 \\
12423.9 \\
12435.5\end{array}$ & $\begin{array}{l}6 p^{3} \mathrm{D}_{1}-6 d^{3} \mathrm{P}_{2}^{\circ} \\
6 s^{3} \mathrm{P}_{1}^{\circ}-6 p^{3} \mathrm{P}_{2} \\
5 d^{3} \mathrm{~F}_{2}^{\circ}-2668\end{array}$ & $\begin{array}{l}-7 \\
-1 \\
0\end{array}$ \\
\hline $\begin{array}{l}8030.5 \\
8007.7 \\
8005.29 \\
7971.02 \\
7910.46\end{array}$ & $\begin{array}{c}100 H l \\
1 h \\
7 h \\
8 h \\
10 h\end{array}$ & $\begin{array}{l}\text { 12449. } 10 \\
12484.5 \\
12488.31 \\
12542.0 \\
12638.02\end{array}$ & $\begin{array}{l}6 s^{3} \mathrm{P}_{2}^{0}-7^{3} \mathrm{P}_{1} \\
5 d^{3} \mathrm{~F}_{3}^{3}-2522 \\
6 s^{3} \mathrm{P}_{2}^{3}-7 p^{3} \mathrm{D}_{2}\end{array}$ & $\begin{array}{l}0 \\
0 \\
0\end{array}$ \\
\hline $\begin{array}{l}7903.52 \\
7863.74 \\
7852.6 \\
7817.2 \\
7808.23\end{array}$ & $\begin{array}{l}2 h \\
8 h \\
2 H \\
4 H \\
10 h\end{array}$ & $\begin{array}{l}\text { 12649. } 12 \\
12713.10 \\
12731.1 \\
12788.8 \\
12803.48\end{array}$ & $\begin{array}{c}5 d^{3} \mathrm{P}_{2}^{\circ}-2759 \\
6 p^{3} \mathrm{D}_{1}-6 d^{3} \mathrm{P}_{1}^{\circ} \\
5 d^{3} \mathrm{D}_{2}^{\circ}-2668\end{array}$ & $\begin{array}{r}+1 \\
-6 \\
0\end{array}$ \\
\hline $\begin{array}{l}7807.0 \\
7801.8 \\
7769.2 \\
7754.97 \\
7741.43\end{array}$ & $\begin{array}{c}2 H \\
8 H \\
3 H \\
100 \\
3\end{array}$ & $\begin{array}{l}12805.5 \\
12814.0 \\
12867.8 \\
12891.41 \\
12913.96\end{array}$ & $\begin{array}{l}5 d^{3} \mathrm{D}_{2}^{\circ}-2666 \\
6 p^{3} \mathrm{D}_{1}-9 s^{3} \mathrm{P}_{1} \\
5 d^{3} \mathrm{D}_{2}^{\circ}-2605 \\
6 s^{3} \mathrm{P}_{1}^{\circ}-6 p^{3} \mathrm{~S}_{1}\end{array}$ & $\begin{array}{r}0 \\
+1 \\
+8 \\
-1\end{array}$ \\
\hline $\begin{array}{l}7719.93 \\
7717.57 \\
7685.30 \\
7627.4 \\
7626.4\end{array}$ & $\begin{array}{c}3 h \\
2 h \\
30 h \\
4 H \\
5 H\end{array}$ & $\begin{array}{l}12949.92 \\
12953.89 \\
13008.28 \\
13107.0 \\
13108.8\end{array}$ & $\begin{array}{c}6 p^{3}{ }^{3} \mathrm{D}_{1}-6 d d^{1} \mathrm{D}_{2}^{\circ} \\
6 s^{1} \mathrm{P}_{1}^{\circ}-7 p{ }^{1} \mathrm{~S}_{0}\end{array}$ & $\begin{array}{l}+1 \\
+1\end{array}$ \\
\hline $\begin{array}{l}7593.85 \\
7530.7 \\
7398.6 \\
6898.50 \\
6462.07\end{array}$ & $\begin{array}{c}2 \\
1 \\
20 H \\
2 \\
4\end{array}$ & $\begin{array}{l}13164.93 \\
13275.3 \\
13512.4 \\
14491.91 \\
15470.65\end{array}$ & $\begin{array}{l}6 s^{3} \mathrm{P}_{0}^{\circ}-6 p^{3} \mathrm{~S}_{1} \\
6 s^{3} \mathrm{P}_{1}^{\circ}-6 p^{1} \mathrm{D}_{2}\end{array}$ & $\begin{array}{r}0 \\
-2 \\
\end{array}$ \\
\hline $\begin{array}{l}6444.85 \\
6354.35 \\
6310.78 \\
6275.75 \\
6203.64\end{array}$ & $\begin{array}{c}5 H \\
40 h \\
100 h l \\
5 h \\
20 h l\end{array}$ & $\begin{array}{l}15512.0 \\
15732.91 \\
15841.53 \\
15929.95 \\
16115.12\end{array}$ & $\begin{array}{l}6 s^{1} \mathrm{P}_{\mathrm{i}}^{\circ}-7 p^{1} \mathrm{P}_{1} \\
6 s^{3} \mathrm{P}_{1}^{\circ}-7 p^{3} \mathrm{D}_{1} \\
6 s^{1} \mathrm{P}_{1}^{\circ}-7 p^{3} \mathrm{P}_{2} \\
6 s^{3} \mathrm{P}_{0}^{\circ}-7 p^{3} \mathrm{D}_{1}\end{array}$ & $\begin{array}{r}0 \\
-1 \\
+1 \\
+1\end{array}$ \\
\hline $\begin{array}{l}6203.01 \\
6171.50 \\
6154.60 \\
6149.71 \\
6110.38\end{array}$ & $\begin{array}{c}3 h \\
150 h \\
200 h l \\
400 h s \\
4 h\end{array}$ & $\begin{array}{l}\text { 16116. } 76 \\
16199.04 \\
16243.52 \\
16256.44 \\
16361.08\end{array}$ & $\begin{array}{l}6 s^{1} \mathrm{P}_{1}^{\circ}-7 p{ }^{3} \mathrm{~S}_{1} \\
6 s^{3} \mathrm{P}_{1}^{\circ}-7 p{ }^{3} \mathrm{P}_{1} \\
6 s^{1} \mathrm{P}_{1}^{\circ}-7 p{ }^{1} \mathrm{D}_{2} \\
6 s^{8} \mathrm{P}_{1}^{\circ}-7 p{ }^{3} \mathrm{D}_{2} \\
6 s^{3} \mathrm{P}_{2}^{\circ}-7 p{ }^{1} \mathrm{P}_{1}\end{array}$ & $\begin{array}{l}+1 \\
-1 \\
0 \\
-2 \\
-1\end{array}$ \\
\hline
\end{tabular}


TABLE 1.-First spectrum of tin (Sn I)-Continued

\begin{tabular}{|c|c|c|c|c|}
\hline$\lambda_{\operatorname{sir}} \mathrm{A}$ & $\begin{array}{l}\text { Intensity and } \\
\text { character }\end{array}$ & $\nu_{\nabla \mathrm{ac}} \mathrm{cm}^{-1}$ & Combination & $\begin{array}{l}\nu_{\text {obs. }}-\mathrm{es} \text { esle. } \\
0.1 \mathrm{~cm}^{-1}\end{array}$ \\
\hline $\begin{array}{l}6073.46 \\
6069.00 \\
6060.75 \\
6054.86 \\
6037.70\end{array}$ & $\begin{array}{l}100 h l \\
250 h s \\
4 h \\
200 h l \\
150 h l\end{array}$ & $\begin{array}{l}16460.53 \\
16472.63 \\
16495.05 \\
16511.10 \\
16558.02\end{array}$ & $\begin{array}{l}6 s^{3} \mathrm{P}_{1}^{\circ}-7 p p^{3} \mathrm{P}_{0} \\
6 s^{3} \mathrm{P}_{0}^{\circ}-7 p p^{3} \mathrm{P}_{1} \\
{ }^{3}{ }^{3} \mathrm{P}_{2}^{\circ}-7 p \\
{ }^{3} \mathrm{D}_{3} \\
6 s^{3} \mathrm{P}_{2}^{\circ}-7 p^{3} \mathrm{P}_{2}\end{array}$ & $\begin{array}{r}-1 \\
+1 \\
0 \\
-1\end{array}$ \\
\hline $\begin{array}{l}6010.96 \\
5970.30 \\
5925.44 \\
5801.81 \\
5761.76\end{array}$ & $\begin{array}{c}4 H \\
100 h \\
50 h l \\
10 h \\
10 h\end{array}$ & $\begin{array}{l}16631.68 \\
16774.95 \\
16871.72 \\
17231.23 \\
17351.01\end{array}$ & $\begin{array}{l}6 s^{3} \mathrm{P}_{2}^{\circ}-7 p{ }^{3} \mathrm{~S}_{1} \\
6 s^{3} \mathrm{P}_{2}^{\circ}-7 p{ }^{1} \mathrm{D}_{2} \\
6 s^{1} \mathrm{P}_{1}^{\circ}-2666 \\
6 s^{3} \mathrm{P}_{1}^{\circ}-7 p{ }^{1}{ }^{1} \mathrm{~s}_{0}\end{array}$ & $\begin{array}{r}0 \\
-1 \\
-2 \\
-1\end{array}$ \\
\hline $\begin{array}{l}5753.59 \\
5631.707 \\
5176.98 \\
5174.54 \\
5160.24\end{array}$ & $\begin{array}{l}15 h \\
500 \\
5 h \\
20 h \\
10 h\end{array}$ & $\begin{array}{l}17375.64 \\
17751.69 \\
19310.92 \\
19320.02 \\
19373.56\end{array}$ & $\begin{array}{l}6 s^{1} \mathrm{P}_{1}^{\circ}-2522 \\
5 p^{1} \mathrm{~S}_{0}-6 s^{3} \mathrm{P}_{0}^{\circ}\end{array}$ & $\begin{array}{l}-2 \\
+1\end{array}$ \\
\hline $\begin{array}{l}\text { 4979. } 73 \\
4931.37 \\
4912.82 \\
\text { 4886. } 36 \\
4766.22\end{array}$ & $\begin{array}{c}20 h \\
10 h \\
3 h \\
2 h \\
5 H\end{array}$ & $\begin{array}{l}20075.83 \\
20272.70 \\
20349.24 \\
20459.44 \\
20975.14\end{array}$ & $\begin{array}{l}6 s^{3} \mathrm{P}_{1}^{\circ}-7 p{ }^{1} \mathrm{P}_{1} \\
6 s^{3} \mathrm{P}_{1}^{\circ}-7 p{ }^{3} \mathrm{P}_{2} \\
6 s^{3} \mathrm{P}_{0}^{\circ}-7 p{ }^{1 \mathrm{P}_{1}} \\
6 s^{3} \mathrm{P}_{1}^{\circ}-7 p p^{3} \mathrm{~S}_{1}\end{array}$ & $\begin{array}{r}0 \\
0 \\
0 \\
-2\end{array}$ \\
\hline $\begin{array}{l}4633.93 \\
4524.744 \\
3801.022 \\
3655.778 \\
3330.620\end{array}$ & $\begin{array}{c}3 h \\
1000 \\
2000 R \\
250 \\
500 r\end{array}$ & $\begin{array}{l}21573.93 \\
22094.52 \\
26301.26 \\
27346.20 \\
30015.84\end{array}$ & $\begin{array}{c}5 p{ }^{1} \mathrm{~S}_{0}-6 s{ }^{1} \mathrm{P}_{\mathrm{i}} \\
5 p{ }^{1} \mathrm{D}_{2}-6 s^{3} \mathrm{P}_{1} \\
5 p{ }^{1} \mathrm{~S}_{0}-5 d^{3} \mathrm{D}_{1}^{\circ} \\
5 p{ }^{1} \mathrm{D}_{2}-6 s^{3} \mathrm{P}_{2}^{\circ}\end{array}$ & $\begin{array}{r}0 \\
+1 \\
0 \\
0\end{array}$ \\
\hline $\begin{array}{l}3262.340 \\
3223.572 \\
3218.707 \\
3175.046 \\
3141.836\end{array}$ & $\begin{array}{c}2500 R \\
10 \\
40 \\
2000 R \\
60\end{array}$ & $\begin{array}{l}30644.04 \\
31012.57 \\
31059.44 \\
31486.53 \\
31819.34\end{array}$ & $\begin{array}{c}5 p p^{1} \mathrm{D}_{2}-6 s^{1} \mathrm{P}_{1} \\
5 p \\
{ }^{1} \mathrm{D}_{2}-195{ }^{2} 9 \\
5 p \\
{ }^{1} \mathrm{~S}_{0}-7 s^{3} \mathrm{P}_{1} \\
5 p{ }^{3} \mathrm{P}_{2}-6 s^{3} \mathrm{P}_{1}^{\circ} \\
5 p{ }^{1} \mathrm{~S}_{0}-5 d^{3} \mathrm{P}_{1}^{\circ}\end{array}$ & $\begin{array}{l}-1 \\
+1 \\
-1 \\
0 \\
-1\end{array}$ \\
\hline $\begin{array}{l}3034.120 \\
3032.805 \\
3009.136 \\
2913.543 \\
2863.324\end{array}$ & $\begin{array}{l}900 R \\
200 h \\
700 R \\
200 h \\
600 R\end{array}$ & $\begin{array}{l}32948.92 \\
32963.21 \\
33222.48 \\
34312.45 \\
34914.22\end{array}$ & $\begin{array}{l}5 p{ }^{3} \mathrm{P}_{1}-6 s^{3} \mathrm{P}_{0}^{\circ} \\
5 p{ }^{1} \mathrm{~S}_{0}-5 d^{1} \mathrm{P}_{1}^{\circ} \\
5 p{ }^{3} \mathrm{P}_{1}-6 s^{3} \mathrm{P}_{1}^{\circ} \\
5 p{ }^{1} \mathrm{~S}_{0}-6 d^{3} \mathrm{D}_{1}^{\circ} \\
5 p{ }^{3} \mathrm{P}_{0}-6 s^{3} \mathrm{P}_{1}^{\circ}\end{array}$ & $\begin{array}{r}-1 \\
-1 \\
+1 \\
0 \\
0\end{array}$ \\
\hline $\begin{array}{l}2850.62 \\
2839.99 \\
2813.575 \\
2812.59 \\
2790.18\end{array}$ & $\begin{array}{l}200 r \\
700 R \\
80 \\
60 \\
4\end{array}$ & $\begin{array}{l}35069.8 \\
35201.1 \\
35531.53 \\
35544.0 \\
35829.4\end{array}$ & $\begin{array}{c}5 p{ }^{1} \mathrm{D}_{2}-5 d^{3} \mathrm{D}_{2}^{\circ} \\
5 p{ }^{3} \mathrm{P}_{2}-6 s^{3} \mathrm{P}_{2}^{\circ} \\
5 p{ }^{1} \mathrm{D}_{2}-5 d^{3} \mathrm{~F}_{2}^{\circ} \\
5 p \\
5{ }^{1} \mathrm{~S}_{0}-7 s^{1} \mathrm{P}_{1}^{\circ} \\
5 p{ }^{3} \mathrm{P}_{2}-6 s^{1} \mathrm{P}_{1}^{\circ}\end{array}$ & $\begin{array}{r}-2 \\
0 \\
-1 \\
-2 \\
0\end{array}$ \\
\hline $\begin{array}{l}2787.96 \\
2785.030 \\
2779.810 \\
2761.78 \\
2744.19\end{array}$ & $\begin{array}{c}60 \\
80 \\
150 \\
20 \\
2 h\end{array}$ & $\begin{array}{l}35858.0 \\
35895.69 \\
35963.09 \\
36197.9 \\
36429.9\end{array}$ & $\begin{array}{c}5 p{ }^{1} \mathrm{~S}_{0}-8 s^{3} \mathrm{P}_{1}^{\circ} \\
5 p{ }^{1} \mathrm{D}_{2}-5 d^{3} \mathrm{D}_{1} \\
5 p \\
5{ }^{1} \mathrm{D}_{2}-5 d^{3} \mathrm{~F}_{3}^{\circ} \\
5 p{ }^{3} \mathrm{P}_{2}-19529 \\
5 p{ }^{1} \mathrm{~S}_{0}+7 d^{3} \mathrm{D}_{1}^{\circ}\end{array}$ & $\begin{array}{r}0 \\
-1 \\
0 \\
+1 \\
+3\end{array}$ \\
\hline $\begin{array}{l}2706.510 \\
2661.243 \\
2636.94 \\
2635.396 \\
2631.28\end{array}$ & $\begin{array}{c}1000 R \\
200 R \\
50 H \\
3 \\
7 H\end{array}$ & $\begin{array}{l}36937.02 \\
37565.27 \\
37911.5 \\
37933.67 \\
37993.0\end{array}$ & $\begin{array}{l}5 p^{3} \mathrm{P}_{1}-6 s^{3} \mathrm{P}_{2} \\
5 p{ }^{3} \mathrm{P}_{1}-6 s^{1} \mathrm{P}_{1}^{\circ} \\
5 p{ }^{1} \mathrm{~S}_{0}-6 d^{3} \mathrm{P}_{1}^{\circ} \\
5 p{ }^{3} \mathrm{P}_{1}-19529 \\
5 p^{1} \mathrm{~S}_{0}-9 s^{3} \mathrm{P}_{1}^{\circ}\end{array}$ & $\begin{array}{r}0 \\
0 \\
+1 \\
0 \\
-6\end{array}$ \\
\hline $\begin{array}{l}2594.424 \\
2588.411 \\
2571.576 \\
2558.01 \\
2548.53\end{array}$ & $\begin{array}{c}200 r \\
7 h \\
500 R \\
40 H \\
5 h\end{array}$ & $\begin{array}{l}38532.69 \\
38622.20 \\
38875.02 \\
39081.2 \\
39226.5\end{array}$ & $\begin{array}{l}5 p^{1} \mathrm{D}_{2}-5 p^{1} \mathrm{D}_{2}^{\circ} \\
5 p^{1} \mathrm{D}_{2}-11919 \\
5 p^{1} \mathrm{D}_{2}-5 d^{3} \mathrm{D}^{\circ} \\
5 p{ }^{1} \mathrm{~S}_{0}-2911 \\
5 p{ }^{1} \mathrm{~S}_{0}-10 s^{3} \mathrm{P}_{1}^{\circ}\end{array}$ & $\begin{array}{r}0 \\
0 \\
0 \\
+1 \\
-2\end{array}$ \\
\hline
\end{tabular}


TABLE 1.-First spectrum of tin (Sn I)-Continued

\begin{tabular}{|c|c|c|c|c|}
\hline$\lambda_{\text {air }} \mathrm{A}$ & $\begin{array}{l}\text { Intensity and } \\
\text { character }\end{array}$ & $\nu_{\mathrm{V}} \mathrm{ac}_{\mathrm{c}} \mathrm{Cm}^{-1}$ & Combination & $\begin{array}{c}\nu_{\text {obs }}-\nu_{\text {oale }} \\
0.1 \mathrm{~cm}^{-1}\end{array}$ \\
\hline $\begin{array}{l}2546.549 \\
2532.87 \\
2531.17 \\
2523.915 \\
2499.11\end{array}$ & $\begin{array}{c}400 r \\
5 H \\
80 H l \\
90 r \\
5 h\end{array}$ & $\begin{array}{l}39257.05 \\
39469.0 \\
39495.6 \\
39609.08 \\
40002.2\end{array}$ & $\begin{array}{r}5 p^{3} \mathrm{P}_{0}-6 s^{1} \mathrm{P}_{1}^{\circ} \\
5 p^{1} \mathrm{~S}_{0}-6 d^{1} \mathrm{P}_{1}^{\circ} \\
5 p^{1} \mathrm{D}_{2}-7 s^{3} \mathrm{P}_{1}^{\circ}\end{array}$ & $\begin{array}{r}0 \\
+2 \\
0\end{array}$ \\
\hline $\begin{array}{l}2495.704 \\
2491.78 \\
2483.392 \\
2476.395 \\
2455.235\end{array}$ & $\begin{array}{c}200 r \\
10 h \\
300 r \\
20 \\
60\end{array}$ & $\begin{array}{l}\text { 40056. } 78 \\
40119.8 \\
40255.35 \\
40369.08 \\
40716.97\end{array}$ & $\begin{array}{c}5 p^{1} \mathrm{D}_{2}-5 d^{3} \mathrm{P}_{2}^{\circ} \\
5 p p^{1} \mathrm{~S}_{0}-8 s^{1} \mathrm{P}_{1}^{\circ} \\
5 p^{3} \mathrm{P}_{2}-5 d^{3} \mathrm{D}_{2}^{\circ} \\
5 p{ }^{1} \mathrm{D}_{2}-5 d^{3} \mathrm{P}_{1}^{\circ} \\
5 p^{3} \mathrm{P}_{2}-5 d^{3} \mathrm{~F}_{2}^{\circ}\end{array}$ & $\begin{array}{r}+2 \\
+1 \\
0 \\
+1 \\
+1\end{array}$ \\
\hline $\begin{array}{l}2433.467 \\
2429.488 \\
2421.697 \\
2408.15 \\
2398.71\end{array}$ & $\begin{array}{c}15 \\
1000 R \\
800 R \\
100 \\
3 h\end{array}$ & $\begin{array}{l}41081.16 \\
41148.44 \\
41280.85 \\
41513.0 \\
41676.4\end{array}$ & $\begin{array}{l}5 p^{3} \mathrm{P}_{2}-5 d^{3} \mathrm{D}_{1}^{\circ} \\
5 p^{3} \mathrm{P}_{2}-5 d^{3} \mathrm{~F}_{3}^{0} \\
5 p^{1} \mathrm{D}_{2}-5 d^{1} \mathrm{~F}_{3}^{\circ} \\
5 p^{1} \mathrm{D}_{2}-5 d^{1} \mathrm{P}_{1}^{\circ}\end{array}$ & $\begin{array}{r}+1 \\
0 \\
0 \\
+1\end{array}$ \\
\hline $\begin{array}{l}2386.96 \\
2380.72 \\
2357.896 \\
2354.837 \\
2334.80\end{array}$ & $\begin{array}{c}10 H w \\
60 \\
20 h \\
1000 R \\
300 R\end{array}$ & $\begin{array}{l}\text { 41881. } 5 \\
41991.3 \\
42397.71 \\
42452.78 \\
42817.1\end{array}$ & $\begin{array}{c}5 p^{3} \mathrm{P}_{1}-5 d^{3} \mathrm{D}_{2}^{\circ} \\
5 p^{1} \mathrm{D}_{2}-6 d^{3} \mathrm{D}_{2}^{\circ} \\
5 p^{3} \mathrm{P}_{1}-5 d^{3} \mathrm{~F}_{2}^{\circ} \\
5 p^{3} \mathrm{P}_{1}-5 d^{3} \mathrm{D}_{1}^{\circ}\end{array}$ & $\begin{array}{r}+1 \\
+2 \\
0 \\
+1\end{array}$ \\
\hline $\begin{array}{l}2332.35 \\
2317.23 \\
2290.66 \\
2286.681 \\
2282.257\end{array}$ & $\begin{array}{c}6 h \\
600 R \\
2 h \\
200 R \\
20\end{array}$ & $\begin{array}{l}42862.0 \\
43141.7 \\
43642.1 \\
43717.97 \\
43802.72\end{array}$ & $\begin{array}{l}5 p^{1} \mathrm{D}_{2}-6 d^{3} \mathrm{D}_{1}^{\circ} \\
5 p^{1} \mathrm{D}_{2}-6 d^{3} \mathrm{~F}_{3}^{\circ} \\
\\
5 p^{3} \mathrm{P}_{2}-5 d^{1} \mathrm{D}_{2}^{\circ} \\
5 p^{1} \mathrm{D}_{2}-7 s^{3} \mathrm{P}_{2}^{\circ}\end{array}$ & $\begin{array}{r}-1 \\
+2 \\
0 \\
0\end{array}$ \\
\hline $\begin{array}{l}2282.01 \\
2268.913 \\
2267.186 \\
2251.168 \\
2246.048\end{array}$ & $\begin{array}{c}2 h \\
400 R \\
30 r \\
60 r \\
400 R\end{array}$ & $\begin{array}{l}43807.5 \\
44060.31 \\
44093.87 \\
44407.59 \\
44508.80\end{array}$ & $\begin{array}{c}5 p^{3} \mathrm{P}_{2}-11919 \\
5 p^{3} \mathrm{P}_{2}-5 d^{3} \mathrm{D}_{3}^{\circ} \\
5 p^{1} \mathrm{D}_{2}-7 s^{1} \mathrm{P}_{1}^{\circ} \\
5 p^{1} \mathrm{D}_{2}-8 s^{3} \mathrm{P}_{1}^{\circ} \\
5 p^{3} \mathrm{P}_{0}-5 d^{3} \mathrm{D}_{1}^{\circ}\end{array}$ & $\begin{array}{r}0 \\
0 \\
+1 \\
0 \\
0\end{array}$ \\
\hline $\begin{array}{l}2233.35 \\
2231.725 \\
2222.555 \\
2220.623 \\
2211.05\end{array}$ & $\begin{array}{c}7 h \\
80 R \\
6 h \\
10 h \\
40 r s\end{array}$ & $\begin{array}{l}44761.8 \\
44794.43 \\
44979.23 \\
45018.35 \\
45213.2\end{array}$ & $\begin{array}{c}5 p^{3} \mathrm{P}_{2}-7 s^{3} \mathrm{P}_{1}^{\circ} \\
5 p^{1} \mathrm{D}_{2}-7 d^{3} \mathrm{D}_{1} \\
5 p{ }^{1} \mathrm{D}_{2}-7 d^{3} \mathrm{~F}_{2}^{\circ} \\
5 p^{1} \mathrm{D}_{2}-7 d^{3} \mathrm{~F}_{3}^{0}\end{array}$ & $\begin{array}{r}0 \\
0 \\
0 \\
+2\end{array}$ \\
\hline $\begin{array}{l}2209.650 \\
2199.337 \\
2194.494 \\
2171.32 \\
2168.522\end{array}$ & $\begin{array}{c}400 R \\
300 R \\
150 R \\
80 r s \\
2\end{array}$ & $\begin{array}{l}45241.88 \\
45454.01 \\
45554.31 \\
46040.4 \\
46099.84\end{array}$ & $\begin{array}{l}5 p^{3} \mathrm{P}_{2}-5 d^{3} \mathrm{P}_{2}^{\circ} \\
5 p^{3} \mathrm{P}_{1}-5 d^{1} \mathrm{D}_{2}^{\circ} \\
5 p^{3} \mathrm{P}_{2}-5 d^{3} \mathrm{P}_{1}^{\circ} \\
5 p{ }^{1} \mathrm{D}_{2}-6 d^{3} \mathrm{P}_{2}^{\circ} \\
5 p p^{1} \mathrm{D}_{2}-4444^{2}\end{array}$ & $\begin{array}{r}0 \\
+1 \\
0 \\
0 \\
0\end{array}$ \\
\hline $\begin{array}{l}2162.982 \\
2151.43 \\
2148.733 \\
2148.460 \\
2147.868\end{array}$ & $\begin{array}{l}5 \\
20 R \\
40 R s \\
15 r s \\
6\end{array}$ & $\begin{array}{l}\text { 46217. } 90 \\
46466.0 \\
46524.36 \\
46530.26 \\
46543.09\end{array}$ & $\begin{array}{l}5 p{ }^{1} \mathrm{D}_{2}-4324 \\
5 p^{3} \mathrm{P}_{2}-5 d^{1} \mathrm{~F}_{3}^{\circ} \\
5 p^{3} \mathrm{P}_{1}-7 s^{3} \mathrm{P}_{0} \\
5 p^{3} \mathrm{P}_{1}-7 s^{3} \mathrm{P}_{1}^{\circ} \\
5 p^{1} \mathrm{D}_{2}-9 s^{3} \mathrm{P}_{1}^{\circ}\end{array}$ & $\begin{array}{r}0 \\
-1 \\
0 \\
0 \\
-1\end{array}$ \\
\hline $\begin{array}{l}2141.434 \\
2140.734 \\
2127.995 \\
2123.62 \\
2121.265\end{array}$ & $\begin{array}{l}20 \\
25 r s \\
7 \\
7 h \\
50 h\end{array}$ & $\begin{array}{l}46682.91 \\
46698.17 \\
46977.68 \\
47074.5 \\
47126.7\end{array}$ & $\begin{array}{c}5 p{ }^{1} \mathrm{D}_{2}-6 d^{1}{ }^{1} \mathrm{D}_{2} \\
5 p{ }^{3} \mathrm{P}_{2}-5 d^{1} \mathrm{P}_{1}^{\circ} \\
5 p{ }^{3} \mathrm{P}_{1}-5 d^{3} \mathrm{P}_{2}^{\circ} \\
5 p{ }^{1} \mathrm{D}_{2}-3467^{2} \\
5 p^{1} \mathrm{D}_{2}-6 d^{3} \mathrm{D}_{3}^{\circ}\end{array}$ & $\begin{array}{r}-2 \\
0 \\
-1 \\
+1 \\
0\end{array}$ \\
\hline $\begin{array}{l}2118.38 \\
2113.93 \\
2100.934 \\
2098.82 \\
2096.39\end{array}$ & $\begin{array}{c}10 h \\
100 R \\
100 \\
3 \\
200 r l\end{array}$ & $\begin{array}{l}47190.9 \\
47290.2 \\
47582.71 \\
47630.6 \\
47685.8\end{array}$ & $\begin{array}{ll}5 p & { }^{1} \mathrm{D}_{2}-3351 \\
5 p & { }^{3} \mathrm{P}_{1}-5 d^{3} \mathrm{P}_{1} \\
5 p & { }^{3} \mathrm{P}_{2}-6 d^{3} \mathrm{D}_{2}^{\circ} \\
5 p & { }^{1} \mathrm{D}_{2}-2911 \\
5 p & { }^{1} \mathrm{D}_{2}-6 d^{1} \mathrm{~F}_{3}^{\circ}\end{array}$ & $\begin{array}{r}+2 \\
0 \\
-1 \\
-1 \\
0\end{array}$ \\
\hline
\end{tabular}


TABLE 1.-First spectrum of tin (Sn I)-Continued

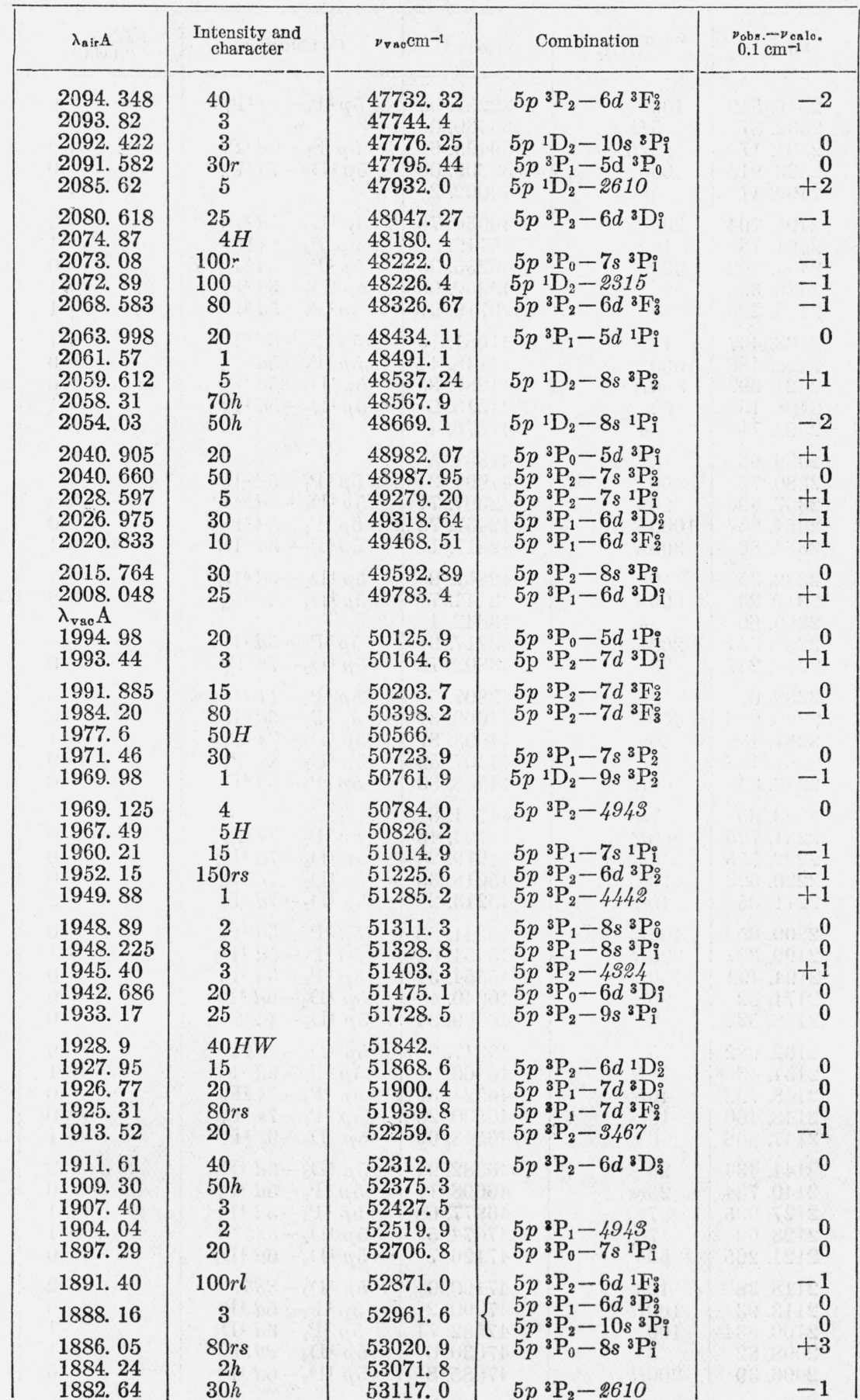


TABLE 1.-First spectrum of tin (Sn I)-Continued

\begin{tabular}{|c|c|c|c|c|}
\hline$\lambda_{\text {air } A}$ & $\begin{array}{l}\text { Intensity and } \\
\text { character }\end{array}$ & $\nu_{\nabla \mathrm{a}_{0} \mathrm{C}} \mathrm{cm}^{-1}$ & Combination & $\begin{array}{l}\nu_{\text {obs. }}-\nu_{\text {oelolo }} \\
0.1 \mathrm{~cm}^{-1}\end{array}$ \\
\hline $\begin{array}{l}\text { 1878. } 63 \\
1873.29 \\
1872.24 \\
1871.29 \\
1870.40\end{array}$ & $\begin{array}{l}5 \\
15 \\
10 h \\
10 \\
1\end{array}$ & $\begin{array}{l}53230.3 \\
53382.1 \\
53411.9 \\
53439.1 \\
53464.5\end{array}$ & $\begin{array}{l}5 p^{3} \mathrm{P}_{2}-6 d^{1} \mathrm{P}_{\mathrm{i}} \\
5 p^{3} \mathrm{P}_{1}-6 d^{3} \mathrm{P}_{1}^{\circ} \\
5 p^{3} \mathrm{P}_{2}-2315 \\
5 p^{3} \mathrm{P}_{1}-9 s^{3} \mathrm{P}_{1}^{\circ}\end{array}$ & $\begin{array}{r}0 \\
-1 \\
+1 \\
+1\end{array}$ \\
\hline $\begin{array}{l}1865.96 \\
1865.52 \\
1862.93 \\
1861.42 \\
1860.32\end{array}$ & $\begin{array}{c}30 r s \\
20 \\
7 h \\
20 \\
200 R s\end{array}$ & $\begin{array}{l}53592.1 \\
53604.3 \\
53679.0 \\
53722.4 \\
53754.2\end{array}$ & $\begin{array}{l}5 p^{3} \mathrm{P}_{0}-7 d^{3} \mathrm{D}_{1}^{\circ} \\
5 p^{3} \mathrm{P}_{1}-6 d^{1} \mathrm{D}_{2}^{\circ} \\
5 p^{3} \mathrm{P}_{2}-8 s^{3} \mathrm{P}_{2}^{\circ}\end{array}$ & $\begin{array}{r}-1 \\
0 \\
0\end{array}$ \\
\hline $\begin{array}{l}1856.82 \\
1854.24 \\
1852.00 \\
1849.49 \\
1848.75\end{array}$ & $\begin{array}{l}2 \\
10 \\
30 \\
10 R \\
50 R\end{array}$ & $\begin{array}{l}53855.5 \\
53930.5 \\
53995.6 \\
54069.0 \\
54090.6\end{array}$ & $\begin{array}{l}5 p^{3} \mathrm{P}_{1}-3467 \\
5 p^{3} \mathrm{P}_{1}-6 d^{3} \mathrm{P}_{0}^{0}\end{array}$ & $\begin{array}{l}0 \\
0\end{array}$ \\
\hline $\begin{array}{l}1848.03 \\
1837.58 \\
1833.08 \\
1829.28 \\
1828.23\end{array}$ & $\begin{array}{l}8+H \\
6 R \\
4 h \\
3 \\
2\end{array}$ & $\begin{array}{l}54111.7 \\
54419.4 \\
54553.0 \\
54666.3 \\
54697.7\end{array}$ & $5 p^{3} \mathrm{P}_{1}-10 s^{3} \mathrm{P}_{1}^{\circ}$ & -2 \\
\hline $\begin{array}{l}\text { 1823. } 00 \\
1819.31 \\
1815.74 \\
1813.04 \\
1804.93\end{array}$ & $\begin{array}{l}120 R s \\
25 \\
40 R \\
30 r \\
10 r\end{array}$ & $\begin{array}{l}54854.6 \\
54965.9 \\
55074.0 \\
55156.0 \\
55403.7\end{array}$ & $\begin{array}{l}5 p^{3} \mathrm{P}_{1}-6 d^{1} \mathrm{P}_{\mathrm{i}} \\
5 p{ }^{3} \mathrm{P}_{0}-6 d^{3} \mathrm{P}_{1}^{\circ} \\
5 p{ }^{3} \mathrm{P}_{0}-9 s^{3} \mathrm{P}_{i} \\
5 p^{3} \mathrm{P}_{1}-11 s^{3} \mathrm{P}_{1}\end{array}$ & $\begin{array}{r}-3 \\
0 \\
-2 \\
+2\end{array}$ \\
\hline $\begin{array}{l}1804.60 \\
1803.16 \\
1795.71 \\
1793.35 \\
1790.75\end{array}$ & $\begin{array}{l}80 \mathrm{Rl} \\
8 \\
3 \\
10 \mathrm{HW} \\
30 \mathrm{Rl}\end{array}$ & $\begin{array}{l}55413.9 \\
55458.1 \\
55688.2 \\
55762 . \\
55842.5\end{array}$ & $5 p^{3} \mathrm{P}_{1}-8 s^{3} \mathrm{P}_{2}^{\circ}$ & -2 \\
\hline $\begin{array}{l}1789.82 \\
1787.38 \\
1780.46 \\
1779.12 \\
1777.90\end{array}$ & $\begin{array}{l}4 R \\
6 \\
5 R \\
R \\
5\end{array}$ & $\begin{array}{l}55871.5 \\
55947.8 \\
56165.3 \\
56207.6 \\
56246.1\end{array}$ & $\begin{array}{l}5 p^{3} \mathrm{P}_{1}-12 s^{3} \mathrm{P}_{1}^{\circ} \\
5 p{ }^{3} \mathrm{P}_{2}-9 s^{3} \mathrm{P}_{2}^{\circ} \\
5 p{ }^{3} \mathrm{P}_{1}-13 s{ }^{3} \mathrm{P}_{1}^{\circ}\end{array}$ & $\begin{array}{l}-2 \\
+5 \\
+4\end{array}$ \\
\hline $\begin{array}{l}1773.40 \\
1764.98 \\
1753.3 \\
1751.46 \\
1745.75\end{array}$ & $\begin{array}{l}20 r s \\
20 R s \\
10 H \\
15 R l \\
3\end{array}$ & $\begin{array}{l}56388.9 \\
56658.0 \\
57035 . \\
57095.2 \\
57282.0\end{array}$ & $\begin{array}{l}5 p{ }^{3} \mathrm{P}_{0}-10 s^{3} \mathrm{P}_{i} \\
5 p{ }^{3} \mathrm{P}_{0}-6 d^{1} \mathrm{P}_{1} \\
5 p{ }^{3} \mathrm{P}_{0}-11 s^{3} \mathrm{P}_{i} \\
5 p^{3} \mathrm{P}_{0}-8 s^{1} \mathrm{P}_{1}^{\circ}\end{array}$ & $\begin{array}{r}-4 \\
0 \\
-1 \\
-3\end{array}$ \\
\hline $\begin{array}{l}\text { 1737. } 21 \\
1733.64 \\
1729.2 \\
1727.15 \\
1719.87\end{array}$ & $\begin{array}{c}10 R l \\
5 h \\
10 H \\
5 R \\
R\end{array}$ & $\begin{array}{l}57563.6 \\
57682.1 \\
57830 . \\
57899.0 \\
58143.9\end{array}$ & $\begin{array}{l}5 p{ }^{3} \mathrm{P}_{0}-12 s^{3} \mathrm{P}_{1}^{\circ} \\
5 p{ }^{3} \mathrm{P}_{1}-9 s^{3} \mathrm{P}_{2}^{\circ} \\
5 p{ }^{3} \mathrm{P}_{0}-13 s^{3} \mathrm{P}_{1} \\
5 p{ }^{3} \mathrm{P}_{0}-14 s^{3} \mathrm{P}_{1}^{\circ}\end{array}$ & $\begin{array}{r}+1 \\
-11 \\
0 \\
0\end{array}$ \\
\hline $\begin{array}{l}1714.54 \\
1714.12 \\
1697.59\end{array}$ & $\begin{array}{r}R \\
R \\
2 h\end{array}$ & $\begin{array}{l}58324.7 \\
58339.0 \\
58907.0\end{array}$ & $\begin{array}{l}5 p^{3} \mathrm{P}_{0}-15 s^{3} \mathrm{P}_{\mathrm{i}}^{\circ} \\
5 p^{3} \mathrm{P}_{1}-10 s^{3} \mathrm{P}_{2}^{\circ}\end{array}$ & $\begin{array}{l}0 \\
0\end{array}$ \\
\hline
\end{tabular}

All lines in table 1 were observed photographically except the first 37 (24738 to $12788 \mathrm{~A}$ ), which are quoted (with corrections) from the radiometric observations of Randall and Wright [7]. These authors stated that the error in their measurements is roughly within $2 \mathrm{~A}$, but the comparison of 20 lines (12535 to $9619 \mathrm{~A}$ ) which both they and I measured shows that their scale is $1.8 \mathrm{~A}$ too large, so this amount was subtracted from each of their values. 
Applying the combination principle as a test of the precision with which relative values of wavelengths are determined, the average residual, $\nu_{\text {obs. }}-\nu_{\text {eale. }}$ of the radiometric observations is $0.8 \mathrm{~cm}^{-1}$, which corresponds approximately to $2 \mathrm{~A}$, as estimated by Randall and Wright. The average residual for lines observed photographically is $0.09 \mathrm{~cm}^{-1}$, which is equivalent to $0.1 \mathrm{~A}$ in the infrared at 11000 $\mathrm{A}, 0.01 \mathrm{~A}$ in the near ultraviolet at $3300 \mathrm{~A}, 0.004 \mathrm{~A}$ at $2000 \mathrm{~A}$, and $0.002 \mathrm{~A}$ at $1700 \mathrm{~A}$. It is no longer necessary to tolerate deviations of 10 or more wave numbers in discussing regularities among ultraviolet tin lines.

Relative intensities shown in column 2 of table 1 begin in the infrared with galvanometer deflections [in brackets] quoted from Randall and Wright [7], who took a deflection of $2,000 \mathrm{~mm}$ as average for the line at $11455 \mathrm{~A}$ and converted other lines to this scale by means of deflection ratios of adjacent lines. These quantitative relative intensities are in italics; the remainder are merely rough averages of the galvanometer deflections taken at different times.

The relative intensities based on visual estimates of the densities and widths of photographic images are subject to progressive errors near the infrared limit of photographic sensitivity; but elsewhere they should be fairly comparable, because variations are more or less averaged out by choosing exposures which give nearly uniform background throughout the spectrum. Besides intensities, column 2 contains some notes on the character of certain lines. When observed with care, the physical appearance of line images is a considerable aid in the analysis of spectral structure. Hazy lines almost invariably indicate that highly excited states are involved, while self-reversal is positive evidence that the line comes from the ground state or from some adjacent metastable state. Dissymmetries have been observed both among hazy and among reversed tin lines. The shading of hazy lines is usually toward longer waves, but occasionally this is reversed. The same is true of self-reversed lines; in some the outer edges shade strongly toward longer waves while others shade toward shorter waves. These effects are probably due to mutual perturbations of adjacent levels of opposite parity [22]. In addition, tin lines are widened by hyperfine structure [23], but the sources and spectrographs used in this investigation would hardly permit detection of this phenomenon. Symbols employed in table 1 for the description of line character are those adopted by the International Astronomical Union [24].

Eighty percent of the observed Sn I lines are classified, the analysis being almost complete for visible and near ultraviolet lines. In the infrared several strong lines (for example, 11277.66, 9850.52, and $9616.40 \mathrm{~A}$ ) remain unclassified, and a half-dozen reversed lines in the extreme ultraviolet $(1860.32,1849.49,1823.00,1804.60,1790.75$, and $1780.46 \mathrm{~A}$ ) have defied classification.

Summarizing the general features of the Sn I spectrum, attention is called to the paucity of visible lines, remarkable concentration of intensity in the near ultraviolet, and comparative richness in the infrared. One line of outstanding intensity, $4524.74 \mathrm{~A}$, overwhelms all others in the visible spectrum and imparts a bluish color to the tin arc flame. The most intense line of the Sn I spectrum is undoubtedly $3262.34 \mathrm{~A}$ in the near ultraviolet, while 3801.02 and $3175.05 \mathrm{~A}$ vie for second place. Since the distribution of lines and intensity in any 
atomic spectrum is a consequence of the atomic-energy states, it is now in order to discuss these aspects of tin atoms.

\section{TERM STRUCTURE OF THE Sn I SPECTRUM}

Tin has atomic number 50 and an electronic structure described as $1 s^{2} 2 s^{2} 2 p^{6} 3 s^{2} 3 p^{6} 3 d^{10} 4 s^{2} 4 p^{6} 4 d^{10} 5 s^{2} 5 p^{2}$. The observed optical spectrum can be (almost) completely accounted for by the two outermost valence electrons, which in various configurations give rise to theoretical spectral terms [25] shown in table 2.

TABLE 2.-Theoretical terms of the Sn I spectrum

\begin{tabular}{|c|c|c|}
\hline $\begin{array}{c}\text { Electron } \\
\text { configurations }\end{array}$ & \multicolumn{2}{|c|}{ Spectral terms } \\
\hline $5 p^{2}$ & \multicolumn{2}{|l|}{${ }^{3} \mathrm{P}_{0},{ }^{3} \mathrm{P}_{1},{ }^{3} \mathrm{P}_{2},{ }^{1} \mathrm{D}_{2},{ }^{1} \mathrm{~S}_{0}$} \\
\hline of lling & $\begin{array}{c}\text { Convergence limit } \\
5 p^{2} \mathrm{P}_{0 \xi \varsigma}^{\circ}(\mathrm{Sn} \mathrm{II})\end{array}$ & $\begin{array}{l}\text { Convergence limit } \\
5 p \cdot{ }^{2} \mathrm{P}_{1 / 3}(\mathrm{Sn} \mathrm{II})\end{array}$ \\
\hline $\begin{array}{ll}5 p & n s \\
5 p & n p \\
5 p & n d \\
5 p & n f\end{array}$ & $\begin{array}{l}{ }^{3} \mathrm{P}_{0}^{\circ},{ }^{3} \mathrm{P}_{1}^{\circ} \\
{ }^{3} \mathrm{D}_{1},{ }^{3} \mathrm{D}_{2},{ }^{3} \mathrm{P}_{0},{ }^{3} \mathrm{P}_{1} \\
{ }^{3} \mathrm{~F}_{2}^{\circ},{ }^{3} \mathrm{~F}_{3},{ }^{3} \mathrm{D}_{1}^{\circ},{ }^{3} \mathrm{D}_{2}^{\circ},{ }^{3} \mathrm{P}_{0}^{\circ},{ }^{3} \mathrm{P}_{1} \\
{ }^{3} \mathrm{G}_{3},{ }^{3} \mathrm{G}_{4},{ }^{3} \mathrm{~F}_{2},{ }^{3} \mathrm{~F}_{3},{ }^{3} \mathrm{D}_{1},{ }^{3} \mathrm{D}_{2}\end{array}$ & $\begin{array}{l}{ }^{3} \mathrm{P}_{2}^{\circ},{ }^{1} \mathrm{P}_{i} \\
{ }^{3} \mathrm{D}_{3},{ }^{3} \mathrm{P}_{2},{ }^{3} \mathrm{~S}_{1},{ }^{1} \mathrm{D}_{2},{ }^{1} \mathrm{P}_{1},{ }^{1} \mathrm{~S}_{0} \\
{ }^{3} \mathrm{~F}_{4}^{\circ},{ }^{3} \mathrm{D}_{3}^{\circ},{ }^{3} \mathrm{P}_{2}^{\circ},{ }^{1} \mathrm{~F}_{3}^{\circ},{ }^{1} \mathrm{D}_{2}, 1{ }^{1} \mathrm{P}_{1} \\
{ }^{3} \mathrm{G}_{5},{ }^{3} \mathrm{~F}_{4},{ }^{3} \mathrm{D}_{3},{ }^{1} \mathrm{G}_{4},{ }^{1} \mathrm{~F}_{3},{ }^{1} \mathrm{D}_{2}\end{array}$ \\
\hline
\end{tabular}

As stated in the introduction, the first significant regularities among arc spectrum lines of tin were discovered in 1894 by Kayser and Runge [3], who found 13 pairs of lines with the frequency difference $5187 \mathrm{~cm}^{-1}$ and 8 pairs separated $1736 \mathrm{~cm}^{-1}$. In 1912 , van Lohuizen [26] attempted to arrange tin lines in series, but the results were purely artificial. No further progress was made until 1918, when Zumstein [27] announced two more constant frequency differences, 1692 and 287 $\mathrm{cm}^{-1}$, only the first of which is real. In 1924, McLennan, Young, and McLay [8] arranged most of the ultraviolet tin lines in a table of combinations with six levels of low energy separated successively by $1692,1735,288,4897$, and $8549 \mathrm{~cm}^{-1}$. The fortuitous differences, 288 and $4897 \mathrm{~cm}^{-1}$, were replaced by their sum, $5185 \mathrm{~cm}^{-1}$, in a paper by H. Sponer [28], who criticized the work of McLennan, et al., on the grounds of large deviations among "constant" differences, numerous double classifications, and discrepancies among intensities and inner quantum numbers of terms proposed as series. In connection with his study of the absorption spectrum of tin vapor in the ultraviolet, Zumstein [9] compiled a table of 49 high levels with 5 low ones, and Sur [29] promptly conjectured the first quantum interpretation of some of these. The identification of five lowest levels with ${ }^{3} \mathrm{P}_{0},{ }^{3} \mathrm{P}_{1},{ }^{3} \mathrm{P}_{2}$, ${ }^{1} \mathrm{D}_{2},{ }^{1} \mathrm{~S}_{0}$ characteristic of a $p^{2}$ configuration was obvious, but the other designations were more or less arbitrary and incorrect. The first real information about the nature of these levels resulted from the Zeemaneffect investigations of Back [10] and of Green and Loring [11], which definitely fixed the quantum numbers of the $5 p^{2}, 5 p 5 d, 5 p 5 s$, and a few more levels. Another attempt at quantum description of tin levels and arrangement in series was made in 1929 by Williams and Charola [30], but their results are largely vitiated by the use of unreliable data 
already cited which tolerates deviations up to $12 \mathrm{~cm}^{-1}$ in relative values of energy levels. An important contribution was made in 1931 by Randall and Wright [7], who observed new lines in the infrared and established some levels of the $5 p 6 p, 5 p 4 f$, and $5 p 5 f$ configurations.

The present investigation, being based on a more complete and accurate description of the Sn I spectrum, has unmasked numerous errors in all preceeding classifications of Sn I lines. Many levels parading in the literature as real are not confirmed, but are replaced by others resting for the most part on combinations which cannot be regarded as fortuitous. In some cases new combinations have corrected the $j$ values of previously known levels.

Besides the levels belonging to $5 p^{2}$, levels from $5 p n s$ have been traced from $n=6$ to $n=15$, the levels (except ${ }^{3} \mathrm{~F}_{4}$ ) from $5 p 5 d$ and $5 p 6 d$ (and fragments of $5 p 7 d$ and $5 p 8 d$ ) have been established, levels from $5 p 6 p$ and $5 p 7 p$ have been revised and completed, while additional levels from $5 p 4 f$ and $5 p 5 f$ have been found.

The $5 p-n s$ combinations are displayed in table 3 , so that the series may be critically examined. The only extended series found, or expected, in the Sn I spectrum are collected here, and will be discussed in more detail below.

TABLE 3.-5p-ns combinations of Sn $\mathbf{I}$

\begin{tabular}{|c|c|c|c|c|c|c|}
\hline \multicolumn{2}{|c|}{$\begin{array}{l}\text { Term symbol } \\
\text { Value }\end{array}$} & \multirow[t]{2}{*}{$\begin{array}{l}5 p^{3} 3 \mathrm{P}_{0} \\
59155.0\end{array}$} & \multirow[t]{2}{*}{$\begin{array}{l}5 p^{2} 3 \mathrm{P}_{1} \\
57463.2\end{array}$} & \multirow[t]{2}{*}{$\begin{array}{l}5 p^{2} 3 \mathrm{P}_{3} \\
55727.3\end{array}$} & \multirow[t]{2}{*}{$\begin{array}{l}5 p^{2} 1 \mathrm{D}, \\
50542.0\end{array}$} & \multirow[t]{2}{*}{$\begin{array}{l}5 p^{3} 1 S_{0} \\
41992.4\end{array}$} \\
\hline Term symbol & Value & & & & & \\
\hline 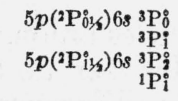 & $\begin{array}{l}24514.2 \\
24240.8 \\
20526.2 \\
19897.9\end{array}$ & $\begin{array}{l}34914.22(600 R) \\
39257.05(400 r)\end{array}$ & $\begin{array}{l}32948.92(900 R) \\
33222.48(700 R) \\
36937.02(1000 R) \\
37565.27(200 R)\end{array}$ & $\begin{array}{l}31486.53(2000 R) \\
35201.1(700 R) \\
35829.4(4)\end{array}$ & $\begin{array}{l}26301.26(2000 R) \\
30015.84(500 r) \\
30644.04(2500 R)\end{array}$ & $\begin{array}{l}17751.69(500) \\
22094.52(1000)\end{array}$ \\
\hline 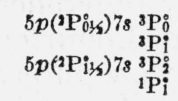 & $\begin{array}{r}10938.8 \\
10932.9 \\
6739.3 \\
6448.2\end{array}$ & $\begin{array}{l}48222.0(100 r) \\
52706.8(20)\end{array}$ & $\begin{array}{l}\text { 46524. 36(40R) } \\
46530.26(15 r) \\
50723.9(30) \\
51014.9(15)\end{array}$ & $\begin{array}{l}44794.43(80 R) \\
48987.95(50) \\
49279.20(5)\end{array}$ & $\begin{array}{l}39609.08(90 r) \\
43802.72(20) \\
44093.87(30 r)\end{array}$ & $\left\{\begin{array}{l}31059.44(40) \\
35544.0(60)\end{array}\right.$ \\
\hline 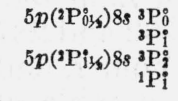 & $\begin{array}{l}6151.9 \\
6194.4 \\
2004.9 \\
1872.7\end{array}$ & $\begin{array}{l}53020.9(80 r) \\
57282.0(3)\end{array}$ & $\begin{array}{l}51311.3(2) \\
51328.8(8) \\
55458.1(8)\end{array}$ & $\begin{array}{l}49592.89(30) \\
53722.4(20)\end{array}$ & $\begin{array}{l}44407.59(60 r) \\
48537.24(5) \\
48669.10(50 h)\end{array}$ & $\begin{array}{l}35858.0(60) \\
40119.8(10 h)\end{array}$ \\
\hline $\begin{array}{l}5 p\left({ }^{2} \mathrm{P}_{036}^{\circ}\right) 9 s{ }^{3} \mathrm{P}_{0}^{0} \\
5 p\left({ }^{3} \mathrm{P}_{1}^{\circ}{ }^{3}\right) 9 s \\
{ }^{3} \mathrm{P}_{2}^{0} \\
{ }^{1} \mathrm{P}_{1}^{0}\end{array}$ & $\begin{array}{c}3998.8 \\
-220 . \\
\end{array}$ & $55156.0(30 r)$ & $\begin{array}{l}53464.5(1) \\
57682.1(5)\end{array}$ & $\begin{array}{l}51728.5(25) \\
55947.8(6)\end{array}$ & $\begin{array}{l}46543.09(6) \\
50761.9(1)\end{array}$ & 37993. $0(7 H l)$ \\
\hline 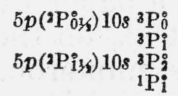 & $\begin{array}{r}2765.7 \\
-1443.8\end{array}$ & $56388.9(20 r)$ & $\begin{array}{l}54697.7(2) \\
58907.0(2)\end{array}$ & $52961.6(3)$ & $47776.25(3)$ & $39226.5(5 h l)$ \\
\hline 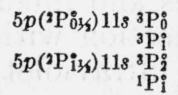 & 2059.7 & $57095.2(15 R)$ & $55403.7(10 r)$ & & & \\
\hline $\begin{array}{l}5 p\left({ }^{3} \mathrm{P}_{036}^{\circ}\right) 12 s s^{3}{ }^{3} \mathrm{P}_{0}^{\circ} \\
{ }^{3} \mathrm{P}_{1}^{0} \\
5 p\left({ }^{3} \mathrm{P}_{1 / 3}{ }^{3}\right) 12 s{ }^{3}{ }^{3}{ }^{1} \mathrm{P}_{1}^{0}\end{array}$ & $\begin{array}{r}1591.5 \\
-5\end{array}$ & $57563.6(10 R)$ & $55871.5(4 R)$ & & & \\
\hline 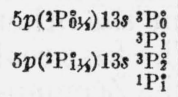 & $\begin{array}{r}1256.0 \\
-\end{array}$ & $57899.0(R)$ & $56207.6(R)$ & & & \\
\hline 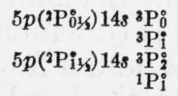 & 1011. & 58143. $9(R)$ & & & & \\
\hline $5 p\left({ }^{2} \mathrm{P}_{035}^{\circ}\right) 15 s{ }^{3}{ }^{3} \mathrm{P}_{\mathrm{i}}^{\dot{3}}$ & 880. & 58324. 7(R) & & & & \\
\hline
\end{tabular}


The $6 s-n p$ combinations are shown in table 4 . Randall and Wright [7] gave ten $6 p$ and four $7 p$ levels; my analysis confirms eight of the former (except $j$ values for 11349 and 10965) and three of the latter (except $j$ value of 7984). Whereas they adopted $\nu=8863$ as $5 p 6 s^{1} \mathrm{P}_{1}^{\circ}-$ $5 p 6 p{ }^{1} \mathrm{~S}_{0}$, I have substituted $\nu=7679$, because it was the outstanding unclassified strong line. Neither assumption is checked by any other combination. No evidence of levels from the $5 p 8 p$ configuration has been found.

TABLE 4. $-6 s-n p$ combinations of Sn I

\begin{tabular}{|c|c|c|c|c|c|}
\hline \multicolumn{2}{|c|}{$\begin{array}{l}\text { Term symbol } \\
\text { Value }\end{array}$} & \multirow[t]{2}{*}{$\begin{array}{l}5 p 6 \mathrm{~s}^{3} \mathrm{P}_{0} \\
24514.2\end{array}$} & \multirow[t]{2}{*}{$\begin{array}{l}5 p 6 s{ }^{8 \mathrm{Pi}} \\
24240.8\end{array}$} & \multirow[t]{2}{*}{$\begin{array}{l}5 p 6 s^{3} \mathrm{P} \mathfrak{2} \\
20526.2\end{array}$} & \multirow[t]{2}{*}{$\begin{array}{l}5 p 6.8{ }^{1} \mathrm{Pi}_{\mathrm{i}} \\
19897.9\end{array}$} \\
\hline Term symbol & Value & & & & \\
\hline 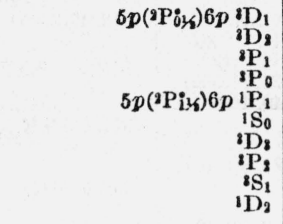 & $\begin{array}{l}16812.7 \\
15916.3 \\
15786.5 \\
15715.0 \\
12551.6 \\
12218.7 ? \\
12148.2 \\
11919.8 \\
11349.3 \\
10965.3\end{array}$ & $\begin{array}{l}\text { 7701. } 0[1870] \\
8727.74[2000] \\
11962.68(80)\end{array}$ & $\begin{array}{l}\text { 7427. } 3[8780] \\
8324.47[480] \\
8454.22[960] \\
8515.72[2580] \\
11689.14(400) \\
\\
12320.86(200) \\
12891.41(100) \\
13275.3(1)\end{array}$ & $\begin{array}{l}\text { 4610. } 0[80] \\
\text { 7974. } 52[425] \\
8377.84[2540] \\
8606.27[2000] \\
9176.85[540] \\
9560.84[250]\end{array}$ & $\begin{array}{l}4109.5[40] \\
7346.5[1440] \\
7679.2[1870] \\
7978.11[425] \\
8548.72[250] \\
8932.63[700]\end{array}$ \\
\hline 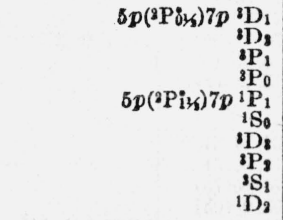 & $\begin{array}{l}8399.2 \\
7994.2 \\
8041.7 \\
7780.2 \\
4165.0 \\
6889.7 \\
4015.1 \\
3968.1 \\
3781.2 \\
3654.4\end{array}$ & $\begin{array}{l}16115.12(20 h l) \\
16472.63(250 h s) \\
20349.24(3 h)\end{array}$ & $\begin{array}{l}15841.53(100 h l) \\
16256.44(400 h s) \\
16199.04(150 h) \\
16460.53(100 h) \\
20075.83(20 h) \\
17351.01(10 h) \\
\\
20272.70(10 h) \\
20459.44(2 h)\end{array}$ & $\begin{array}{l}\text { 12127. } 0(1 h) \\
12542.00(8 h) \\
12484.5(1 h) \\
16361.08(4 h) \\
16511.10(200 h l) \\
16558.02(150 h l) \\
16744.95(100 h) \\
16871.72(50 h l)\end{array}$ & $\begin{array}{l}11498.86(5 h) \\
11913.82(20 h) \\
11856.13(5 h) \\
12117.84(8 h) \\
15732.91(40 h) \\
13008.28(30 h) \\
15929.95(5 h) \\
1616.76(3 h) \\
16243.52(200 h)\end{array}$ \\
\hline
\end{tabular}

All $5 p-n d$ combinations are presented in table 5 . The $6 d$ levels were tentatively selected by comparing relative positions and combining properties with those of the well-established $5 d$ levels. There are, however, some intensity anomalies which may be due to perturbations of other levels. Because of almost complete overlapping of $6 d, 7 d$, and $8 d$ configurations, and the fragmentary character of the higher terms, it seems presumptuous to attempt grouping these higher levels into terms and series.

Table 5 also shows combinations of two superfluous levels at 19529 and $11919 \mathrm{~cm}^{-1}$, which possibly belong to the $5 s 5 p^{3}$ configuration.

Unfortunately it is still impossible to establish all expected levels from the $5 p n f$ configurations, principally on account of insufficient infrared data. Their strongest combinations range in wave number from 2000 to $13000 \mathrm{~cm}^{-1}$, part of which range has never been explored, and faint lines are unrecorded in most of the remainder. Randall and Wright [7] reported no galvanometer deflections less than $30 \mathrm{~mm}$. Inability at the present time to complete the full sets of levels due to $5 p n f$ curbs the possibility of establishing $5 p 5 d^{3} \mathrm{~F}_{4}$; its $j$ value is too large to permit combination with any $5 p^{2}$ levels. Randall and Wright [7] reported some $n s-n f$ combinations, but no evidence for such combinations is found among my data. 
TABLE 5.-5p-nd combinations of Sn $\mathrm{I}$

\begin{tabular}{|c|c|c|c|c|c|c|}
\hline \multicolumn{2}{|c|}{$\begin{array}{l}\text { Term symbol. } \\
\text { Value }\end{array}$} & \multirow[t]{2}{*}{$\begin{array}{l}5 p^{2}{ }^{3} \mathrm{P}_{0} \\
59155.0\end{array}$} & \multirow[t]{2}{*}{$\begin{array}{l}5 p^{2}{ }^{3} \mathrm{P}_{1} \\
57463.2\end{array}$} & \multirow[t]{2}{*}{$\begin{array}{l}5 p^{2}{ }^{3} \mathrm{P}_{2} \\
55727.3\end{array}$} & \multirow[t]{2}{*}{$\begin{array}{l}5 p^{2}{ }^{1} \mathrm{D}_{2} \\
50542.0\end{array}$} & \multirow[t]{2}{*}{$\begin{array}{l}5 p^{2}{ }^{1 \mathrm{~S}_{0}} \\
41992.4\end{array}$} \\
\hline Term symbol & Value & & & & & \\
\hline 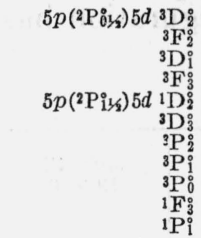 & $\begin{array}{r}15472.0 \\
15010.4 \\
14646.2 \\
14578.9 \\
12009.3 \\
11667.0 \\
10485.4 \\
10173.0 \\
9667.8 \\
9261.2 \\
9029.1\end{array}$ & $44508.80(400 R)$ & $\begin{array}{l}41991.3(60) \\
42452.78(1000 R) \\
42817.1(300 R) \\
45454.01(300 R) \\
46977.68(7) \\
47290.2(100 R) \\
47795.44(30 r) \\
48434.11(20)\end{array}$ & $\begin{array}{l}\text { 40255. 35(300r) } \\
\text { 40716. } 97(60) \\
41081.16(15) \\
41148.44(1000 R) \\
43717.97(200 R) \\
44060.31(400 R) \\
45241.88(400 R) \\
45554.31(150 R) \\
4646.0(20 R) \\
46698.17(25 r)\end{array}$ & \begin{tabular}{|l}
$35069.8(200 r)$ \\
$35531.53(80)$ \\
$35895.69(80)$ \\
$35963.09(150)$ \\
$38532.69(200 R)$ \\
$38875.02(500 R)$ \\
$40056.78(200 r)$ \\
$40369.08(20)$ \\
\\
$41230.81(800 R)$ \\
$41513.0(100)$
\end{tabular} & $27346.20(250)$ \\
\hline 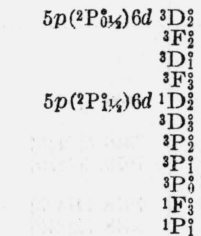 & $\begin{array}{l}8144.5 \\
7994.8 \\
7679.9 \\
7400.5 \\
3858.9 \\
341.9 \\
4501.6 \\
4081.0 \\
3972.6 \\
2856.2 \\
2497.0\end{array}$ & $55074.0(40 R)$ & $\begin{array}{l}49318.64(30) \\
49468.51(10) \\
49783.42(25) \\
\\
53604.3(20) \\
\\
52961.6(3) \\
53382.1(15) \\
54090.6(30 R) \\
54965.9(25)\end{array}$ & $\begin{array}{l}47582.71(100) \\
47732.32(40) \\
48047.27(25) \\
48326.67(80 r) \\
51868.6(15) \\
52312.0(40) \\
51225.6(150 r) \\
\\
52871.0(100 r) \\
53230.3(5)\end{array}$ & $\begin{array}{l}42397.71(20 h) \\
42861.95(6 h) \\
43141.7(600 R) \\
46682.91(20) \\
47126.74(50 h) \\
46040.4(80 \mathrm{r}) \\
\\
47685.8(200 r)\end{array}$ & $37911.5(50 H)$ \\
\hline $\begin{array}{r}5 s 5 p^{3} ? 2 \\
5 p\left({ }^{2} \mathrm{P}_{03 / 3}^{\circ}\right) 7 d{ }^{3}{ }^{3} \mathrm{D}_{1}^{\circ} \\
{ }^{3} \mathrm{~F}_{2}^{\circ} \\
5 p 7 d \\
\text { and } \\
5 p 8 d\end{array}$ & \begin{tabular}{|r|}
19529.5 \\
11919.8 \\
5562.8 \\
5523.6 \\
5329.0 \\
4943.8 \\
4442.2 \\
4324.1 \\
3467.6 \\
3851.3 \\
2911.3 \\
2610.2 \\
2315.5
\end{tabular} & 53592.1(30r) & $\begin{array}{l}37933.67(3) \\
51900.4(20) \\
51939.8(80 r) \\
52519.9(2)\end{array}$ & $\begin{array}{l}36197.9(20) \\
43807.5(2) \\
50164.6(3) \\
50203.7(15) \\
50398.2(80) \\
50784.0(4) \\
\\
51285.2(1) \\
51403.3(3) \\
52259.6(20) \\
\\
53117.0(30 h) \\
53411.9(10 h)\end{array}$ & $\begin{array}{l}31012.57(10) \\
38622.20(7) \\
44979.23(6 h) \\
45018.35(10 h) \\
45213.2(40 r) \\
\\
46099.84(2) \\
46217.90(5) \\
47074.50(7 h) \\
47190.9(10 h) \\
47630.6(3) \\
47932.0(5) \\
48226.4(100 h)\end{array}$ & $39081.2(40 H)$ \\
\hline
\end{tabular}

\section{SPECTRAL SERIES AND IONIZATION POTENTIAL}

Since law-abiding spectral series are always of special interest and importance for determinations of spectroscopic ionization potentials and for tracing the convergence of term sequences in one spectrum to limits in the next, we shall now return to the $5 p-n s$ combinations. Any $s$ electron reacting with $5 p$ gives rise to four energy levels, ${ }^{1} \mathrm{P}^{\circ},{ }^{3} \mathrm{P}_{0}^{\circ},{ }^{3} \mathrm{P}_{1}^{\circ},{ }^{3} \mathrm{P}_{2}^{\circ}$, which with increasing $n$ converge toward the ground state of the ion represented by $5 p^{2} \mathrm{P}_{0 \%, 11 / 2}^{0}$. Furthermore, as shown in table 2 , two of the levels $\left({ }^{3} \mathrm{P}_{0}\right.$ and $\left.{ }^{3} \mathrm{P}_{1}\right)$ end on ${ }^{2} \mathrm{P}_{01 / 2}^{\circ}$, while the remaining two $\left({ }^{1} \mathrm{P}_{1}\right.$ and $\left.{ }^{3} \mathrm{P}_{2}\right)$ end on ${ }^{2} \mathrm{P}_{13 / 2}^{\circ}$. The ${ }^{2} \mathrm{P}$ term describing the normal state of a tin ion has a level separation of $4252 \mathrm{~cm}^{-1}$, according to Green and Loring [11] or Narayan and Rao [31], and this large interval divides the levels belonging to any configuration with a running electron into two widely separated groups.

As a general rule, the most prominent and longest series of lines or terms in any spectrum is the series which involves the normal state of the atom (or ion) and produces lines which are easily self-reversed, or observed in pure absorption. This rule is obeyed by the Sn I spectrum, in which I have succeeded in tracing the sequence of $n s{ }^{3} \mathrm{P}_{1}$ levels for 10 values of $n$ from $n=6$ to $n=15$. The tail end of this series was observed only in absorption, the last three lines appearing as reversals in the continuous background on the spectrograms. 
Although the $n s^{3} \mathrm{P}_{2}$ levels have larger $j$ values (usually accompanied by stronger combinations), they cannot combine with the ground level, ${ }^{3} \mathrm{P}_{0}$, because $\Delta j=2$, and combinations with the first metastable state, ${ }^{3} \mathrm{P}_{1}$, cannot be followed beyond five members, that is, from $n=6$ to $n=10$. The $n s^{3} \mathrm{P}_{0}$ levels can combine only with ${ }^{3} \mathrm{P}_{1}$, and the fourth line could hardly be expected when the intensities of the first three are observed to be in the ratio 900,40,2. A rapid intensity decrement also limits the intersystem series $5 p^{3} \mathrm{P}_{0}-n s^{1} \mathrm{P}_{1}$ to three lines with rolative intensities $400,20,3$.

A Ritz formula of the type

$$
\nu=L-R /\left(n+a+\frac{b}{n^{2}}\right)^{2},
$$

in which $R_{\mathrm{sn}}=109737.0$, has been calculated to represent each of the above-mentioned series. The series $5 p^{3} \mathrm{P}_{0}-n s^{3} \mathrm{P}_{1}^{\circ}$ is satisfactorily reproduced by the formula

$$
\nu=59160-109737 /\left(n-3.64395-\frac{8.22715}{n^{2}}\right)^{2}, n=6,7,8, \ldots
$$

The "observed minus calculated" wave numbers for 10 successive lines are $-2,-142,+1,-29,+17,+1,-3,+6,+15,+20$. In other words, the average error in wavelength representation of nine lines is $\pm 0.4 \mathrm{~A}$, and the calculated position of the second line is $6.2 \mathrm{~A}$ too short. This raises a question concerning the identification of two levels, 10932 and 10173 , designated $7 s^{3} \mathrm{P}_{1}^{\circ}$ and $5 d^{3} \mathrm{P}_{1}^{\circ}$, respectively, by Green and Loring [11], but the reverse by Back [10]. Both attached question marks to their designations of the latter level, but present evidence favors the former interpretation. If the latter were correct, the calculated position of the second series line would be $27 \mathrm{~A}$ too short and there would be an additional intensity anomaly.

The series $5 p^{3} \mathrm{P}_{1}-n s^{3} \mathrm{P}_{2}^{\circ}$ is represented by the formula

$$
\nu=61703-109737 /\left(n-3.68077-\frac{7.72844}{n^{2}}\right)^{2}, n=6,7,8, \ldots
$$

with the following "observed minus calculated" wave numbers for five successive lines: $+10, \pm 0,-19, \pm 0,+20$. This series converges toward ${ }^{2} \mathrm{P}_{11 / 2}^{\circ}$ of $\mathrm{Sn}$ II ; and since $5 p^{3} \mathrm{P}_{1}$ is removed $1692 \mathrm{~cm}^{-1}$ from $5 p^{3} \mathrm{P}_{0}$, this amount must be added to the limit to obtain the energy difference $5 p^{2.3} \mathrm{P}_{0}(\mathrm{Sn} \mathrm{I})-5 p \cdot{ }^{2} \mathrm{P}_{1 / 2}^{0}(\mathrm{Sn} \mathrm{II})=61703+1692=63395 \mathrm{~cm}^{-1}$.

The Ritz formula

$$
\nu=57640-109737 /\left(n-3.76466-\frac{4.57780}{n^{2}}\right)^{2}, n=6,7,8 \text {, }
$$

fits the three-line series $5 p^{3} \mathrm{P}_{1}-n s^{3} \mathrm{P}_{0}^{0}$ perfectly. Since $5 p^{3} \mathrm{P}_{1}$ is 1692 $\mathrm{cm}^{-1}$ from $5 p^{3} \mathrm{P}_{0}$, an absolute value of $57640+1692=59332$ is indicated for the latter.

Likewise, the Ritz formula

$$
\nu=63286-109737 /\left(n-3.54774-\frac{11.35288}{n^{2}}\right)^{2}, n=6,7,8,
$$

represents without error the three-line series $5 p^{3} \mathrm{P}_{0}-n s^{1} \mathrm{P}_{1}^{\circ}$. The limit, 63286, is a measure of the energy difference $5 p^{2 \cdot 3} \mathrm{P}_{0}$ 
(Sn I) $-5 p \cdot{ }^{2} \mathrm{P}_{113}^{\circ}$ (Sn II), to be compared with 63395 obtained from the five-line series, $5 p^{3} \mathrm{P}_{1}-n s^{3} \mathrm{P}_{2}^{\circ}$. Regarding the latter twice as reliable as the former, we obtain a weighted mean of 63360 ; and subtracting the doublet separation of the Sn II ground state, we obtain 63360$4252=59107 \mathrm{~cm}^{-1}$ as the absolute value of $5 p^{3} \mathrm{P}_{0}$.

By assigning three times as much weight to the 10-line series, $5 p^{3} \mathrm{P}_{0}-n s^{3} \mathrm{P}_{1}$, as to the three-line series, $5 p^{3} \mathrm{P}_{1}-n s^{3} \mathrm{P}_{0}^{\circ}$, we obtain 59203 $\mathrm{cm}^{-1}$ as the absolute value of $5 p^{3} \mathrm{P}_{0}$. Finally, the average of 59107 and 59203 is $59155 \mathrm{~cm}^{-1}$, which is the value I have adopted as the basis for all Sn I terms. This is almost identical with 59160 given by the 10 term series. After this value, 59155, was adopted, I noticed that in 1924 McLennan, et al. [8], by assuming that the frequencies 54098, 54428,54672 , and 54852 formed a Rydberg series with values of $m$ equal to $8,9,10$, and 11 arrived at a value of 59158 for this constant. This must be regarded as a most remarkable coincidence, because the assumed series is entirely fictitious, none of the levels being confirmed.

The only subsequent attempts to derive the absolute value of Sn I terms were made by Green and Loring [11] in 1927 and by Randall and Wright [7] in 1931. The former correctly classified the first three lines of the series $5 p^{3} \mathrm{P}_{0}-n s{ }^{3} \mathrm{P}_{1}$, and the value of $5 p^{3} \mathrm{P}_{0}$ was computed approximately as $59690 \mathrm{~cm}^{-1}$. Randall and Wright assumed that two unidentified levels (6891 and $4387 \mathrm{~cm}^{-1}$ in my list) represented $5 p\left({ }^{2} \mathrm{P}_{01 / 2}^{\circ}\right) 4 f$ and $5 p\left({ }^{2} \mathrm{P}_{01 / 2}\right) 5 f$ terms in a Rydberg series and thus obtained $59192 \mathrm{~cm}^{-1}$ for the absolute value of the lowest level of Sn I.

The value 59155 for $5 p^{2}{ }^{3} \mathrm{P}_{0}$ (SnI) corresponds to an ionization potential of $1.2336 \times 10^{-4} \times 59155=7.297$ volts. If the series electron is driven to the higher level of the $\mathrm{Sn}^{+}$ion, the potential necessary for ionization will be $1.2336 \times 10^{-4} \times(59155+4252)=7.822$ volts.

An impression of the reliability of the limits derived from the various $n s$ sequences may perhaps be obtained from a comparison of 59203 and 63360 . Since the former is given by two series converging to ${ }^{2} \mathrm{P}_{0 \frac{1}{2}}$ (Sn II) and the latter by two series converging to ${ }^{2} \mathrm{P}_{1 \% / 2}^{\circ}$ (Sn II), the difference, $4157 \mathrm{~cm}^{-1}$, is a measure of the level separation in the ${ }^{2} \mathrm{P}$ term representing the normal state of ionized tin atoms. The true value of this separation is $4252 \mathrm{~cm}^{-1}$ as stated before [11,31].

All the SnI levels and terms that I have been able to establish and interpret with reasonable certainty are exhibited in table 6 . Rather than arrange the levels in strict order of magnitude and thereby mix up a variety of terms and configurations, I have preferred to group the levels primarily under configurations and secondarily in order of energy. So far as possible like levels from similar configurations are repeated in the same order so that they may be easily compared. Intervals between levels of triplet terms are shown in column 4. The "level separation" opposite any particular level expresses the distance in $\mathrm{cm}^{-1}$ from that level to the one with unit greater $j$ value. It will be noticed that many of these level separations are of the order of $4000 \mathrm{~cm}^{-1}$, suggestive of the two ionic states toward which all Sn I series converge. Indeed, it is the 4252 $\mathrm{cm}^{-1}$ wide doublet of SnII which divides into two groups all Sn I levels belonging to any configuration with a running electron. Because this convergence interval is larger than any interval between successive terms (beyond the second), it results in a confusing inter- 
lacing or overlapping of terms probably entailing perturbations of intervals, intensities, and magnetic splitting factors, thus destroying all simple criteria of term designations. When there is such promiscuity between coupled electrons, the begotten terms will have difficulty in recognizing their parents. In the presence of such superposition, although numerous transitions between levels are theoretically possible, many of the resulting lines are probably in the still unexplored infrared, and the levels themselves cannot be established except through the lines associated with them.

TABLE 6.-Levels and terms of Sn I

\begin{tabular}{|c|c|c|c|}
\hline Electron configuration & Term symbol & Term value $\mathrm{cm}^{-1}$ & Level separation \\
\hline \multirow[t]{4}{*}{$5 p^{2}$} & ${ }^{3} \mathrm{P}_{0}$ & 59155.0 & 1691. 8 \\
\hline & ${ }^{3} \mathrm{P}_{1}$ & 57463. 2 & 1735.9 \\
\hline & ${ }^{3} \mathrm{P}_{2}$ & 55727.3 & \\
\hline & ${ }_{1 \mathrm{~S}_{0}}$ & $\begin{array}{l}50542.0 \\
41992,4\end{array}$ & \\
\hline \multirow[t]{2}{*}{$5 p\left({ }^{2} \mathrm{P}_{03 s}\right) 6 s$} & ${ }^{3} \mathrm{P}_{0}^{\circ}$ & 24514.2 & 273. 4 \\
\hline & ${ }^{3} \mathbf{P}_{\mathbf{i}}$ & 24240.8 & 3714.6 \\
\hline $5 p\left({ }^{2} \mathrm{P}_{13 s}^{\circ}\right) 6 s$ & ${ }^{3} \mathrm{P}_{2}^{\circ}$ & 20526.2 & \\
\hline \multirow{3}{*}{$5 p\left({ }^{2} \mathrm{P}_{036}\right) 6 p$} & ${ }^{3} \mathrm{D}_{1}$ & 16812.7 & 896.4 \\
\hline & ${ }^{3} \mathrm{D}_{2}$ & 15916.3 & 3768.1 \\
\hline & ${ }^{3} \mathrm{P}_{1}$ & 15786.5 & 3866. 7 \\
\hline \multirow{5}{*}{$5 p\left({ }^{2} \mathbf{P}_{136}\right) 6 p$} & ${ }^{3} \mathrm{P}_{0}$ & 15725. 0 & -61.5 \\
\hline & $\begin{array}{l}{ }_{1}^{1} P_{1} \\
{ }_{1} S_{0}\end{array}$ & $\begin{array}{l}12551.6 \\
12218.7 ?\end{array}$ & \\
\hline & ${ }^{3} \mathrm{D}_{3}$ & 12148. 2 & \\
\hline & ${ }^{3} \mathrm{P}_{2}$ & 11919.8 & \\
\hline & $\begin{array}{l}{ }^{3} \mathrm{~S}_{1} \\
{ }^{1} \mathrm{D}_{2}\end{array}$ & $\begin{array}{l}11349.3 \\
10965.3\end{array}$ & \\
\hline \multirow[t]{4}{*}{$5 p\left({ }^{2} \mathrm{P}_{035}^{\circ}\right) 5 d$} & ${ }^{2} \mathrm{D}_{2}^{2}$ & 15472.0 & 3805.0 \\
\hline & ${ }^{3} \mathrm{~F}_{2}^{\circ}$ & 15010.4 & 431. 5 \\
\hline & ${ }^{3} \mathrm{D}_{\mathrm{i}}^{\circ}$ & 14646.2 & -825.8 \\
\hline & ${ }^{3} \mathrm{~F}_{3}^{\circ}$ & 14578.9 & \\
\hline \multirow{5}{*}{$5 p\left({ }^{2} \mathrm{P}_{136}\right) 5 d$} & ${ }^{1} \mathrm{D}_{2}^{\circ}$ & 12009.3 & \\
\hline & $\begin{array}{l}{ }^{3} \mathrm{D}_{3}^{\circ} \\
{ }^{3} \mathrm{P}_{0}^{\circ}\end{array}$ & $1166 \% .0$ & \\
\hline & ${ }^{3} \mathbf{P}_{i}^{2}$ & 10173.0 & -312.4 \\
\hline & ${ }^{3} \mathrm{P}_{0}^{\circ}$ & 9667.8 & -495.2 \\
\hline & ${ }^{1} \mathrm{P}_{1}$ & $\begin{array}{l}9201.2 \\
9029.1\end{array}$ & \\
\hline \multirow[t]{2}{*}{$5 p\left({ }^{2} \mathrm{P}_{035}\right) 7 s$} & ${ }^{3} \mathrm{P}_{0}^{1}$ & 10938.8 & 5.9 \\
\hline & ${ }^{3} \mathrm{P}_{\mathrm{i}}$ & 10932. 9 & 4193. 6 \\
\hline $5 p\left({ }^{2} \mathrm{P}_{136}\right) 7 s$ & ${ }^{3} \mathrm{P}_{2}^{\circ}$ & 6739. 3 & \\
\hline \multirow{3}{*}{$5 p\left({ }^{2} \mathbf{P}_{036}^{\circ}\right) 7 p$} & ${ }^{1} \mathrm{P}_{i}$ & $\begin{array}{l}6448.2 \\
83992\end{array}$ & 415. 0 \\
\hline & ${ }^{3} \mathrm{D}_{2}$ & 7984. 2 & 3969. 1 \\
\hline & ${ }^{3} \mathbf{P}_{1}$ & 8041. 7 & 4073. 6 \\
\hline \multirow{5}{*}{$5 p\left({ }^{2} \mathrm{P}_{\mathrm{i}}{ }^{16}\right) 7 p$} & ${ }^{3} \mathbf{P}_{0}$ & 7780.2 & -619.0 \\
\hline & ${ }^{1} P_{1}$ & 4165. 0 & \\
\hline & $\begin{array}{l}{ }^{1} \mathrm{~S}_{0} \\
{ }^{3} \mathrm{D}_{3}\end{array}$ & $\begin{array}{l}\text { 6889. } 7 \\
4015.1\end{array}$ & \\
\hline & ${ }^{3} \mathbf{P}_{2}$ & 3968.1 & \\
\hline & ${ }^{3} \mathrm{~S}_{1}$ & 3781. 2 & \\
\hline \multirow{4}{*}{$5 p\left({ }^{2} \mathrm{P}_{0 y / 4}^{\circ}\right) 6 d$} & $\begin{array}{l}1 \mathrm{D}_{2} \\
3 \mathrm{D}^{2}\end{array}$ & 3604.4 & $4779 ?$ \\
\hline & ${ }^{3} \mathrm{~F}_{2}^{2}$ & 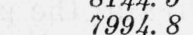 & $\begin{array}{r}594.3 \\
5\end{array}$ \\
\hline & ${ }^{3} \mathrm{D}_{\mathrm{i}}$ & 7679.9 & -464.6 \\
\hline & ${ }^{3} \mathrm{~F}_{3}^{0}$ & 7400.5 & \\
\hline
\end{tabular}


TABLE 6.-Levels and terms of Sn I-Continued

\begin{tabular}{|c|c|c|c|}
\hline Electron configuration & Term symbol & Term value $\mathrm{cm}^{-1}$ & Level separation \\
\hline 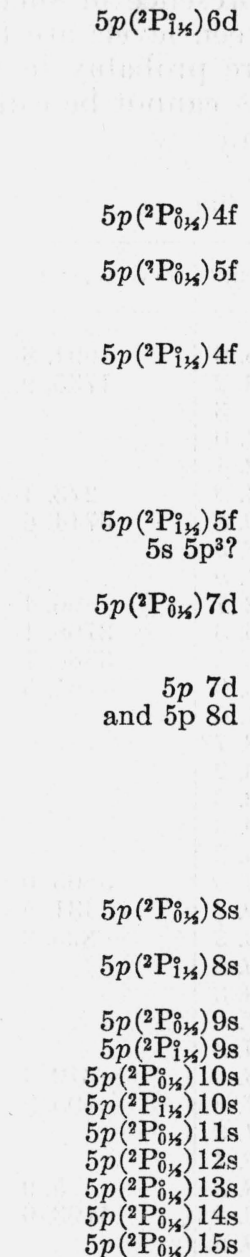 & 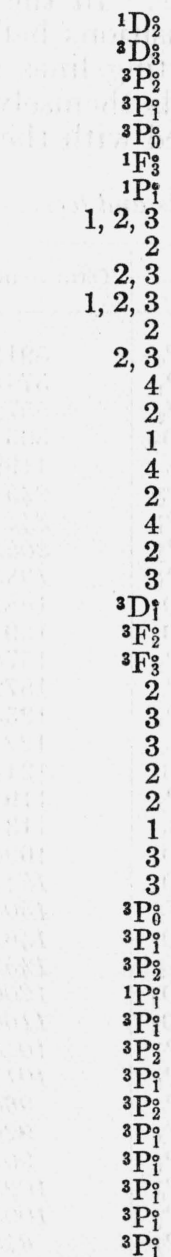 & $\begin{array}{r}3858.9 \\
3415.3 \\
4501.6 \\
4081.0 \\
3372.6 ? \\
2856.2 \\
2497.0 \\
6906.0 \\
6891.2 \\
4392.4 \\
4387.3 \\
4383.2 \\
2759.0 \\
2754.3 \\
2668.5 \\
2666.5 \\
2605.0 \\
2522.1 \\
108.4 \\
19529.5 \\
11919.8 \\
5562.8 \\
5523.6 \\
5329.0 \\
4943.3 \\
4442.2 \\
4324.1 \\
3467.6 \\
3351.3 \\
2911.3 \\
2610.8 \\
2315.5 \\
6151.9 \\
6134.4 \\
2004.9 \\
1872.7 \\
3998.8 \\
-220.0 \\
2765.9 \\
-1443.8 \\
2059.9 \\
1591.5 \\
1256.0 \\
1011.1 \\
830.3\end{array}$ & $\begin{array}{r}17.5 \\
4129.5 \\
\\
4219 . \\
4209.5\end{array}$ \\
\hline
\end{tabular}

Another consequence of the wide doublet to which Sn I terms converge is that high terms going to the upper limit may exceed the lower ionization energy and become negative. Several negative terms are listed near the end of table 6 . It was shown above that a potential of 7.297 volts measures the energy required to move a valence electron from its normal state in a neutral Sn atom to the lowest energy state of the $\mathrm{Sn}^{+}$ion, and that ionization to the higher (metastable) ionic state increases the potential to 7.822 volts. The former value, 7.297 volts, expresses the principal ionization potential of tin. 


\section{REFERENCES}

[1] W. F. Meggers, J. Opt. Soc. Am. 29, 258 (1939).

[2] H. Kayser, Handbuch der Spectroscopie 6, 509 (1912).

[3] H. Kayser and C. Runge, Wiedem. Ann. 52, 93 (1894).

[4] R. Arnolds, Z. wiss. Phot. 13, 313 (1914).

[5] F. M. Walters, Jr., BS Sci. Pap. 17, 174 (1921) S411.

[6] H. M. Randall, Astrophys. J. 34, 1 (1911).

[7] H. M. Randall and N. Wright, Phys. Rev. 38, 457 (1931).

[8] J. C. McLennan, J. F. T. Young, and A. B. McLay, Trans. Roy. Soc. Can. 18, 57 (1924).

[9] R. V. Zumstein, Phys. Rev. 27, 150 (1926).

[10] E. Back, Z. Physik 43, 309 (1927).

[11] J. B. Green and R. A. Loring, Phys. Rev. 30, 574 (1927).

[12] A. G. Shenstone, Phil. Trans. Roy. Soc. (London) [A] 237, 453 (1938).

[13] W. F. Meggers and K. Burns, BS Sci. Pap. 18, 185 (1922) S441.

[14] R. W. Wood, Nature, 140, 723 (1937).

[15] Eastman Kodak Co., Photographic Plates for Use in Spectroscopy and Astronomy (1935).

[16] Proc. Sixth Summer Conference on Spectroscopy, p. 114 (John Wiley \& Sons, New York, N. Y., 1939).

[17] K. Burns and F. M. Walters, Jr., Pub. Allegheny Obs. 6, 159 (1929); 8, 39 (1931); 8, 27 (1931).

[18] J. C. Boyce and H. A. Robinson, J. Opt. Soc. Am. 26, 133 (1936).

[19] W. F. Meggers, J. Research NBS 14, 33 (1935) RP755; W. F. Meggers and C. J. Humphreys, J. Research NBS 18, 543 (1937) RP992.

[20] H. Kayser, Tabelle der Schwingungszahlen (Julius Springer, Berlin, 1927).

[21] W. F. Meggers and C. G. Peters, Bul. BS 14, 697 (1918) S327.

[22] T. L. deBruin, C. J. Humphreys and W. F. Meggers, BS J. Research 11, 417 (1933) RP599.

[23] H. Schüler and H. Westmeyer, Naturwissenschaften 21, 660 (1933); S. Tolansky, Proc. Roy. Soc. (London) [A] 144, 574 (1934).

[24] Trans. Int. Astron. Union 6, 100 (1938).

[25] F. Hund, Linienspektren und Periodisches System der Elemente (Julius Springer, Berlin, 1927).

[26] J. van Lohuizen, Z. wiss. Phot. 11, 397 (1912).

[27] R. V. Zumstein, Trans. Roy. Soc. Can. 12, 59 (1918).

[28] H. Sponer, Z. Physik 32, 19 (1925).

[29] N. K. Sur, Z. Physik 41, 791 (1927).

[30] A. T. Williams and F. Charola, J. phys. 9, 377 (1929).

[31] A. L. Narayan and K. R. Rao, Z. Physik 45, 350 (1927).

Washington, November 30, 1939. 